\title{
Let the Experts Decide? Asymmetric Information, Abstention, and Coordination in Standing Committees ${ }^{1}$
}

\author{
Rebecca Morton ${ }^{2} \quad$ Jean-Robert Tyran ${ }^{3}$
}

November 2, 2008

\footnotetext{
${ }^{1}$ We appreciate greatly the work of Michael Rudy Schrøder, Lars Markan Reenberg, Nils Bruhn Christensen, and Marco Piovesan for excellent research support.

${ }^{2}$ Department of Politics, NYU, 19 West 4th Street, 2nd Floor, New York, NY 10012, USA. Email: rebecca.morton@nyu.edu.

${ }^{3}$ Department of Economics, University of Copenhagen, Studiestraede 6, 1455 Copenhagen, Denmark
} 


\begin{abstract}
We examine abstention when voters in standing committees are asymmetrically informed and there are multiple pure strategy equilibria-swing voter's curse (SVC) equilibria where voters with low quality information abstain and equilibria when all participants vote their information. When the asymmetry in information quality is large, we find that voting groups largely coordinate on the SVC equilibrium which is also Pareto Optimal. However, we find that when the asymmetry in information quality is not large and the Pareto Optimal equilibrium is for all to participate, significant numbers of voters with low quality information abstain. Furthermore, we find that information asymmetry induces voters with low quality information to coordinate on a non-equilibrium outcome. This suggests that coordination on "letting the experts" decide is a likely voting norm that sometimes validates SVC equilibrium predictions but other times does not.
\end{abstract}


Individuals make binary decisions by majority voting in many contexts from elections to legislatures to city councils to faculty department meetings to juries. A central question in the literature on formal models of voting has been the extent that majority voting leads to information aggregation when participants have private information but all would like to choose the same outcome as if they had complete information as posited by Condorcet (1785) . ${ }^{1}$ Yet, in most of this work the possible abstention of voters is ignored. This makes sense for one of the principal applications of these models, that is, juries, since abstention is not allowed. But it does not make sense for many of the other voting situations. Abstention or simply not showing up for votes is allowed in most elections, legislatures, city councils, and faculty department meetings.

Furthermore, one might argue that a norm in many of these voting situations is to delegate decisions to "the experts" or those individuals known to have expertise about a matter. For example, suppose an issue before a city council is whether to construct a new sewage plant. We can imagine that some of the city council members will have greater knowledge about the merits of the decision than others and that this will be known because they come from different business backgrounds or parts of the city or are on particular subcommittees. Alternatively, when a faculty department votes on whether to hire a new member, we can imagine that some members have greater knowledge of the individual's merits than others, and this heterogeneity in information will be known. We particularly expect this to be true in standing committees such as legislatures, city councils, and faculty departments since the same individuals repeatedly interact in voting situations over a series of sequential choices and are likely to know the overall qualities of each others' information.

In a seminal set of papers, Feddersen and Pesendorfer (1996, 1999), hereafter FP, incorporate abstention into voting situations with asymmetric information and demonstrate that such delegation to experts can be rational even when the cost of voting is zero. The reasoning is that a voter's choice only matters if he or she is pivotal. But if an uninformed voter is pivotal, then

\footnotetext{
${ }^{1}$ See for example Austen-Smith and Banks (1996), Feddersen and Pesendorfer (1998), and Meirowitz (2002).
} 
that implies that he or she may cancel out the vote of a more informed voter who has similar preferences. Thus, voting would be "cursed" for this individual, and the individual should rationally abstain. Feddersen and Pesendorfer's model has been labeled the "swing voter's curse," hereafter SVC. The prediction that uninformed voters will abstain and delegate their votes to informed voters has been supported in laboratory elections by Battaglini, Morton, and Palfrey (2008a,b), hereafter BMP.

BMP investigate two situations in which information quality is binary. In one situation voters are either fully informed or uninformed and in the other voters are either fully informed or somewhat informed. In BMP somewhat or less informed voters are ones who have some prior information that one outcome is better than the other, but not full information about the best outcome. BMP also consider treatments where some voters are partisans and always vote for a particular choice regardless of their information. In general, in BMP, both uninformed and less informed voters abstain and delegate their votes to informed voters when it is theoretically optimal for them to do so. However, there is more error on the part of less informed voters. That is, some less informed voters do participate and vote for the choice that their information leads them to believe is optimal.

Yet, there are features of the formal setup of the BMP experiments that are at variance with some observational worlds of voting with abstention. First, in the BMP experiments voters do not know for sure whether other voters are more informed or not, just the probability that they are more informed, which is the same for all voters. The uncertainty is over the actual number of informed voters in the electorate. This might make sense when thinking of a large election. But as noted above in many standing committee voting situations we would expect voters to know that some voters have access to better quality of information. This difference may matter to voters in such groups where knowing for certain that some voters are informed can lead them to be more likely to abstain and delegate votes than when the number of informed voters is unknown. 
Second, BMP evaluate only a special case of the SVC model where there is always a probability that some voters are fully informed. A more interesting case would be where no voter is perfectly informed, but some voters have access to better quality of information, which still may be imperfect. ${ }^{2}$ When no voter has perfect information, multiple equilibria can exist in pure strategies. That is, it is possible that equilibria exist as in SVC, where only the voters with high quality information participate, but also equilibria exist where all voters participate across information quality levels. Thus, in cases where multiple equilibria exist, the less informed voters face strategic uncertainty over whether they should either vote their information or abstain, depending on their expectations of what other similar voters will be choosing. Furthermore, which equilibrium is Pareto Optimal (i.e. results in all voters receiving higher utility levels) depends on the difference in informational quality. If the difference in information quality is not too large, then voters' utilities are higher in the equilibrium where all participate rather than in the SVC equilibrium, but if the difference in information quality is large, then voters' utilities are higher in the SVC equilibrium.

In this paper we consider these important cases that are more likely to capture voting in standing committees. We find significant support for the SVC equilibrium predictions when no voter is fully informed and there is a large degree of information asymmetry such that the SVC equilibrium is Pareto Optimal. However, we find that in some cases where the Pareto Optimal equilibrium is for all voters to participate even though information asymmetry exists, significant numbers of voters coordinate instead on the SVC equilibrium. The information asymmetry leads voters to overvalue the advantage of voters with higher quality information, experts, and to coordinate on the inferior SVC equilibrium. This evidence suggests that the tendency of less informed voters to delegate their votes can be strong and that abstaining when less informed may occur even when a Pareto Optimal equilibrium with all voters participating exists. We find that the tendency to delegate to more informed voters is so strong that groups sometimes

\footnotetext{
${ }^{2}$ McMurray (2008) theoretically considers when the quality of information differs across voters and the implications for large elections.
} 
coordinate on a non-equilibrium strategy combination that resembles SVC equilibria but is not a Bayesian Nash equilibrium. The behavior of voters suggests that they are following a norm of "letting the experts decide" even when that norm is not an equilibrium prediction. Our results then demonstrate that this norm can lead voters to make choices that are suboptimal. In the next section we present our model of abstention with asymmetric noisy information which is the basis for our experimental analysis and the theoretical predictions for our treatments. In Section III we discuss our experimental procedures and present our experimental results. Section IV summarizes and addresses implications of our analysis for future research on information aggregation in voting.

\section{A Model of Abstention with Known Asymmetric Information Qualities}

\section{Basic Setup}

We consider a voting game with a finite number of participants, $n \geq 3$. Participants choose whether to vote for one of two options, $a$ or $b$, or abstain. The option that receives a majority of the votes is declared the winner and ties are broken randomly. There are two states of the world $A$ and $B$. The probability that state $A$ occurs is given by $1>\pi \geq 0.5$. Voters have homogenous preferences. That is, all voters have the same utility function. We normalize voters' utility to equal 1 if either option $a$ is selected in state of the world $A$ or $b$ is chosen in state of the world $B$, and 0 otherwise. ${ }^{3}$

Voter $i$ receives an imperfect signal of the world, $\sigma_{i} \in\{a, b\}$. There are two types of voters, those who receive high quality signals and those that receive low quality signals. Define $p$ as the probability that a voter with high quality signals receives an $a$ signal when the state of the world is $A$ and a $b$ signal when the state of the world is $B$ and $q$ as the probability that a voter with low quality signals receives an $a$ signal when the state of the world is $A$ and a $b$ signal when

\footnotetext{
${ }^{3}$ Although we assume that voters have the same preferences over the final outcome, we can think of their information as resulting in different observed preferences over intermediate policies. Others have similarly modeled voters in elections in this fashion, see for example Canes-Wrone, Herron, and Shotts (2001).
} 
the state of the world is $B$. Thus, the probability that a voter with high quality signals receives an $a$ signal when the state of the world is $B$ and a $b$ signal when the state of the world is $A$ is given by $1-p$ and $1-q$ is similarly defined for voters with low quality signals. We assume that $1>p \geq q>0.5$. Define $n^{H}$ as the number of voters who receive high quality signals; thus the number who receive low quality signals is given by $n-n^{H}$. Finally, and importantly, we assume that these probabilities and numbers of voters of each type are common knowledge.

Our general setup allows both pure strategy equilibria where all participate, even when information quality varies, and ones where only those voters with high quality signals participate, as in the swing voter's curse model. To see how this is true, in the next section we consider the special case where $n=3, n^{H}=1, n^{L}=2$, and $\pi=0.5$, which is one of the cases we used in the experiments.

\section{Equilibria in the Three Voter Game}

\section{All Vote Equilibria}

In solving for the voting equilibria, we assume that voters condition their vote choice on being pivotal. We also assume that if voters vote, they vote their signals. We solve for the pure strategy Bayesian-Nash equilibria to this game under these assumptions. First, we examine whether an equilibriaum exists where no one votes. In this case, any voter can decide the outcome and all votes are potentially pivotal. The expected utility from not voting for each voter given others' abstention is equal 0.5 since the election is a tie, but the expected utility from voting for each given others' abstention is equal to the probability of making a correct decision which is $p$ for voters with high quality information and $q$ for voters with low quality information. Since both $p$ and $q$ are greater than 0.5 , it cannot be an equilibrium for all voters to abstain.

Second, we investigate whether an equilibrium exists where everyone votes. Since we have an odd number of voters, in the case of everyone voting, there is only one pivotal event in the absence of one's vote, a tie. So voters' choices of whether to vote or not are conditioned on 
their being a tie vote if they choose not to participate. A voter has his or her own signal as information, but a voter also potentially has information conveyed in the event of a pivotal vote. This is the crucial insight of the FP model.

Consider the voter with high quality information who has received an $a$ signal. Without loss of generality we label this voter as voter $H$ and the two voters with low quality signals as voters $L 1$ and $L 2$. Voter $H$ 's vote only matters if voters $L 1$ and $L 2$ 's votes are tied which would occur if one gets an $a$ signal and the other has a $b$ signal. Label this event $P I V^{H}$. Voter $H$ compares her utility from abstaining to voting conditioned on this pivotal event. If voter $H$ abstains, in the pivotal event she receives an expected utility of 0.5 since the outcome of the election would be a tie and $a$ and $b$ are equally likely to win.

Label $E U_{H}\left(\right.$ All Vote $\left.\mid \sigma_{H}=a, P I V^{H}\right)$ voter $H$ 's expected utility of voting when $L 1$ and $L 2$ participate given the pivotal event. $E U_{H}\left(\right.$ All Vote $\left.\mid \sigma_{H}=a, P I V^{H}\right)$ is a function then of the likelihood that $A$ is the true state of the world conditioned on $H$ 's signal and the pivotal event as follows:

$$
E U_{H}\left(\text { All Vote } \mid \sigma_{H}=a, P I V^{H}\right)=\operatorname{Pr}\left(A \mid \sigma_{H}=a, P I V^{H}\right) * 1+\operatorname{Pr}\left(B \mid \sigma_{H}=a, P I V^{H}\right) * 0
$$

From Bayes Rule, the expected utility then is equal to the probability that $A$ is the true state of the world given that the high quality voter gets an a signal and the two low quality voters' signals are split. Furthermore, this expected utility can be shown to simply equal $p$ when $\pi=0.5$ :

$$
\begin{aligned}
E U_{H}\left(\text { All Vote } \mid \sigma_{H}=a, P I V^{H}\right) & =\operatorname{Pr}\left(A \mid \sigma_{H}=a, P I V^{H}\right) \\
& =\frac{\operatorname{Pr}\left(\sigma_{H}=a, P I V^{H} \mid A\right) \pi}{\operatorname{Pr}\left(\sigma_{H}=a, P I V^{H} \mid A\right) \pi+\operatorname{Pr}\left(\sigma_{H}=a, P I V^{H} \mid B\right)(1-\pi)} \\
& =\frac{2 p q(1-q) 0.5}{2 p q(1-q) 0.5+2(1-p) q(1-q) 0.5}=p
\end{aligned}
$$

Since $p>0.5$, voter $H$ should participate and vote for $a$. Similarly, if voter $H$ receives a $b$ signal, 
he or she should vote for $b$.

Now consider the voters with low quality information. Take voter $L 1$ and assume he or she has received an a signal. Voter $L 1$ 's vote only matters if the election is a tie without his or her vote, so either voter $H$ has an $a$ signal and voter $L 2$ has a $b$ signal or vice-versa. Call this pivotal event $P I V^{L}$. As with voter $H$, if voter $L 1$ abstains, in the pivotal event the election is a tie and voter $L 1$ 's expected utility is 0.5 .

Similarly, as with voter $H$, voter $L 1$ 's expected utility if he or she votes for $a$ in the pivotal event is given by the probability that the true state of the world equals $A$ in the pivotal event. Furthermore, from Bayes' Rule this expected utility can be shown to equal $q$ when $\pi=0.5$ :

$$
\begin{aligned}
E U_{L 1}\left(\text { All Vote } \mid \sigma_{L 1}=a, P I V^{L}\right) & =\operatorname{Pr}\left(A \mid \sigma_{L 1}=a, P I V^{L}\right) \\
& =\frac{\operatorname{Pr}\left(\sigma_{L 2}=a, P I V^{L} \mid A\right) \pi}{\operatorname{Pr}\left(\sigma_{L 1}=a, P I V^{L} \mid A\right) \pi+\operatorname{Pr}\left(\sigma_{L 1}=a, P I V^{L} \mid B\right)(1-\pi)} \\
& =\frac{\left(p q(1-q)+(1-p) q^{2}\right) 0.5}{\left(p q(1-q)+(1-p) q^{2}\right) 0.5+\left((1-p) q(1-q)+p(1-q)^{2}\right) 0.5} \\
& =q
\end{aligned}
$$

As with voter $H$, since $q>0.5$, voter 2 should vote for $a$. Similarly, if voter $L 1$ receives a $b$ signal he or she should vote for $b$. The case of voter $L 2$ is analogous. Thus, an equilibrium exists in which all voters vote their signals in this case. In the rest of the paper we will label this type of equilibrium an All Vote Equilibrium. It is also straightforward to show that no equilibrium exists in which only the voters with low quality information participate since in that case the voter with high quality information, voter $H$, has an incentive to vote as we have seen above.

\section{Swing Voter's Curse Equilibria}

Now we examine whether equilibria exists in which only the voter with high quality information, voter $H$, participates. We know from the analysis above that if the two voters with low quality information are abstaining, the optimal response for voter $H$ is to vote his or her signal. What remains is to determine if it is an optimal response for the two voters with low quality information 
to abstain given that voter $H$ is participating.

Suppose voter $L 1$ receives an $a$ signal. Since only voter $H$ is participating, voter $L 1$ 's vote is pivotal only if that vote is different from voter $H$ 's, in which case voter $L 1$ will force a tie election and voter $L 1$ 's utility is equal is equal to 0.5 . What happens if $L 1$ abstains? In the pivotal event when $L 1$ 's signal differs from $H, H$ will decide the election. So $L 1$ 's expected utility in the pivotal event is the probability that $H$ 's signal is correct in the pivotal event. Given that $L 1$ has received an $a$ signal, the pivotal event is that $H$ has received a $b$ signal.

$$
\begin{aligned}
E U_{L 1}\left(\mathrm{SVC} \mid \sigma_{H}=b \& \sigma_{L 1}=a\right) & =\operatorname{Pr}\left(B \mid \sigma_{H}=b \& \sigma_{L 1}=a\right) \\
& =\frac{p(1-q)}{p(1-q)+(1-p) q}
\end{aligned}
$$

It is straightforward to show that $E U_{L 1}\left(\operatorname{SVC} \mid \sigma_{H}=b \& \sigma_{L 1}=a\right)=0.5$ if $p=q$, and is greater than 0.5 if $p>q$. Thus, it is an optimal response for $L 1$ to abstain if $H$ is voting his or her signal and $L 2$ is abstaining since $H$ has better quality information. Similarly, we can show that voter $L 2$ 's optimal response is to abstain as well. Thus a swing voter's curse equilibria is possible. We will label this equilibrium the SVC equilibrium.

Finally, note that there are no asymmetric equilibria in which the two voters with low quality information choose different pure strategies. As we have seen voter $H$ always votes. And, given that voter $H$ is voting if one voter with low quality information has an optimal response to vote, so does the other voter with low quality information. Such an All Vote equilibrium always exists. Furthermore, in the SVC equilibrium both voters with low quality information optimally abstain. Thus voters with low quality information face strategic uncertainty since they would prefer to coordinate on the same actions, either voting or nonvoting.

\section{Probability of Correct Decisions and Pareto Optimality}

To determine the relative informational efficiency of the two types of equilibria, we calculate the probability that the majority votes correctly in the two possible equilibria; the equilibrium 
where all vote and the SVC equilibrium. Assuming the true state of the world is $A$, then in the All Vote equilibrium the probability that the majority votes correctly is equal to the probability that at least two of the three voters receive an $a$ signal which is given by (since everyone votes, there are no tie elections):

$$
\operatorname{Pr}(\text { Majority Correct Decision })=2 p q(1-q)+q^{2}
$$

In contrast, in the SVC equilibrium, the probability that the majority votes correctly is simply equal to the probability that voter $H$ has received a correct signal, which is $p$. Thus when $\frac{q^{2}}{(1-2 q(1-q))}>p$, the Pareto Optimal equilibrium is the All Vote equilibrium and when $\frac{q^{2}}{(1-2 q(1-q))}<p$ the Pareto Optimal equilibrium is the SVC case. The two equilibria are equivalent in optimality when $\frac{q^{2}}{(1-2 q(1-q))}=p$. Figure 1 illustrates how Pareto Optimality varies with the values of $p$ and $q$. The dotted lines mark the boundary of the region where $1>p \geq q>0.5$ and the solid line represents the values of $p$ and $q$ such that $\frac{q^{2}}{(1-2 q(1-q))}=p$. Above the solid line are values of $p$ and $q$ in which the All Vote Equilibrium is Pareto Optimal and below the solid line are values of $p$ and $q$ in which the SVC Equilibrium is Pareto Optimal.

Figure 1

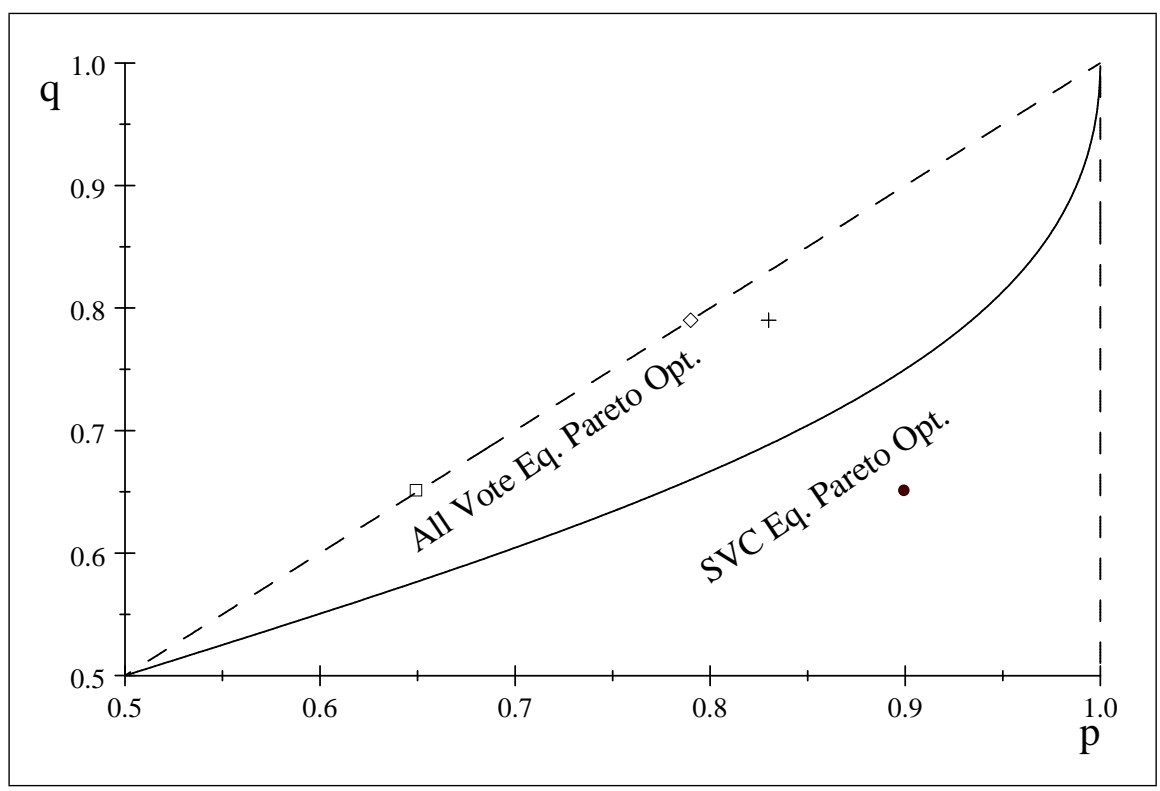


Also, Figure 1 illustrates the values of $p$ and $q$ that we use in four of the treatments in the experiment. The dot represents the point $(p, q)=(.9, .65)$, which we label the ABS treatment since the SVC equilibrium is Pareto Optimal in this case. The cross represents the point $(p, q)=$ $(.83, .79)$, which we label the VOT treatment since the All Vote equilibrium is Pareto Optimal in that treatment. By comparing behavior in the ABS and VOT treatments, we can determine the extent that the desire to delegate to experts in situations of known asymmetric information qualities lead voters to make suboptimal decisions.

We also use two treatments where $p=q$ and the values of $q$ are equivalent to those used in the ABS and VOT treatments, respectively, which are also illustrated in the figure. The square represents the point $(p, q)=(.65, .65)$, which we label the HOM65 treatment and the diamond represents the point $(p, q)=(.79, .79)$, which we label the HOM79 treatment. In both of these treatments an All Vote equilibrium exists, following the reasoning above and it is clearly Pareto Optimal. Although perhaps not technically SVC equilibria since all voters have the same quality of information, equilibria also exist in which only one voter votes. To see how this might be true, assume that there are three voters whose information quality is given by $q$, which we call $L 1, L 2$, and $L 3$. Assume that $L 1$ is voting and $L 2$ is abstaining. Should $L 3$ vote? Voter $L 3$ 's vote will be pivotal if her signal differs from $L 1$ in which case she or he will cause a tie election and receive an expected utility of 0.5. But if she or he abstains, his or her expected utility (in the pivotal event) is also equal to 0.5 because with conflicting signals both states of the world are equally likely. Therefore, $L 3$ is indifferent between voting and abstaining. Similarly it is rational for either only $L 2$ or only $L 3$ to participate. Thus, there are three possible SVC equilibria in both HOM65 and HOM79. Of course, these SVC equilibria involve choosing the weakly dominated strategy of abstaining and significant coordination between voters as to which single voter will participate. Furthermore, the behavior in these equilibria are not supported by a norm of "letting the experts decide" since all voters have the same quality of information.

We include these two homogeneous treatments so that we can compare the voters with low 
quality information in treatments $\mathrm{ABS}$ and VOT with the behavior of voters with the same levels of information but where the quality is homogeneous. That is, we can compare behavior of voters with low quality information in ABS with all voters in HOM65 and behavior of voters with low quality information in VOT with all voters in HOM79.

\section{Voters with High Quality Information and Coordination}

We also consider a fifth treatment, which we label VOTB, also with $(p, q)=(.83, .79)$. The difference between VOT and VOTB is that in VOTB there is only one voter with low quality information but two voters with high quality information. Thus, we examine a case where voters with low quality information do not appear to face a coordination problem. However, the predictions from this treatment are not necessarily simple as we explore. First, as the analysis above shows, an equilibrium exists in VOTB where all voters participate since in the pivotal event their expected utility from voting equals either $p$ or $q$ and both are greater than 0.5 , the expected utility from not voting in the pivotal event.

Now we turn to whether an SVC equilibrium exists in this treatment. Without loss of generality we label the two voters with high quality information $H 1$ and $H 2$ respectively, and the voter with low quality information, $L$. Consider the choice of voter $L$. Assume that both $H 1$ and $H 2$ are voting their signals. The pivotal event for $L$ will be when $H 1$ and $H 2$ 's signals conflict. In which case, $L$, would break a tie. If $L$ chooses not to vote in this case, his or her expected utility is equal to 0.5 . If he or she chooses to vote his or her expected utility is simply equal to his or her information quality, $q$, as shown in equation 6 below:

$$
E U_{L 2}\left(\sigma_{L}=a \& P I V^{L}\right)=\frac{q p(1-p)}{q p(1-p)+(1-q) p(1-p)}=q
$$

Since $q>0.5$, the voter with low quality information should always participate when the two voters with high quality information are participating. Is it optimal for both $H 1$ and $H 2$ to participate? Suppose both $L$ and $H 1$ are voting. Should $H 2$ vote? In the pivotal event, $L$ and $H 1$ have conflicting signals. If $H 2$ abstains, then his or her expected utility is 0.5. But 
following the analysis above, if $H 2$ votes, his or her expected utility from voting is $p$. Thus, given that $L$ is voting, both $H 1$ and $H 2$ should vote. Hence, a traditional SVC equilibrium in which all voters with high quality information vote and those with low quality information abstain does not exist for any values of $p$ and $q$. Note that this implies as well that behavior where the voter with low quality information abstains and "lets the experts decide" is not an equilibrium norm. Thus, VOTB is a strong test of whether voters with low quality information are drawn to the norm of letting voters with higher quality information decide even when doing so involves out of equilibrium behavior.

Note that in VOTB, though, as in HOM65 and HOM79, SVC like equilibria exist in which only one voter with high quality information participates. To see this, suppose that $H 1$ is voting and $L$ is abstaining. Should $H 2$ vote? In the pivotal event, $H 2$ has received a different signal from $H 1$ and by voting will cause a tie election. The expected utility for $H 2$ from voting is thus equal to 0.5. However, as above in HOM65 and HOM79, the expected utility for $\mathrm{H} 2$ in the pivotal event is also equal to 0.5 , and $H 2$ is indifferent between voting or not. Therefore, abstention is a rational response of $H 2$ in this case. Voter $L$ should also abstain if $H 1$ is voting but $H 2$ is not, since the expected utility to $L$ from voting is also equal to 0.5 ,but the expected utility of abstaining is given by equation 4 above and as long as $p>q, L$ 's expected utility from abstaining, delegating his or her vote to $H 1$ is higher than 0.5 . Thus, even in this treatment two SVC equilibria exist-one in which only $H 1$ participates and one in which only $H 2$ participates. As in HOM65 and HOM79, these SVC equilibria involve using weakly dominated strategies and significant coordination of $H 1$ and $H 2$ on who votes and who abstains.

What about the Pareto Optimality of these three possible equilibria in treatment VOTB? In the All Vote equilibrium the probability that the majority votes correctly is equal to the probability that at least two of the three voters receive a correct signal which is given by (since everyone votes, there are no tie elections):

$$
\operatorname{Pr}(\text { Majority Correct Decision })=p^{2}+2 p q(1-p)
$$


As above in the two SVC equilibria, the probability that the majority votes correctly is simply equal to the probability that voter $H$ has received a correct signal, which is $p$. However, it is straightforward to show that given that $0.5 \leq q \leq p<1$, then the All Vote equilibrium Pareto dominates both SVC equilibria.

\section{Summary of Theoretical Predictions}

Table 1 summarizes our treatments and the associated theoretical predictions. Treatments ABS and VOT are our two primary treatments. In both of these treatments there exist an SVC equilibrium and an All Vote equilibrium. Thus voters face strategic uncertainty. In the ABS treatment, the SVC equilibrium is Pareto Optimal, while in the VOT treatment, the All Vote equilibrium is Pareto Optimal. If voters coordinate on Pareto Optimality, we expect then that in ABS subjects will coordinate on the SVC equilibrium and in VOT subjects will coordinate on the All Vote equilibrium. Notice that the expected utility from these two treatments is exactly symmetric by design and thus the benefits from coordination on the predicted Pareto Optimal equilibrium is exactly the same for both treatments.

\begin{tabular}{|c|c|c|c|c|c|c|c|}
\hline \multicolumn{9}{|c|}{ Table 1: Summary of Treatments and Predictions } \\
\hline \multicolumn{9}{|c|}{ Treatment } & $p$ & $q$ & $H$ & $L$ & All Vote & SVC & Norm \\
\hline Primary & & & & & & & \\
\hline ABS & 0.90 & 0.65 & 1 & 2 & 0.83 & 0.90 & 0.90 \\
\hline VOT & 0.83 & 0.79 & 1 & 2 & 0.90 & 0.83 & 0.83 \\
\hline Secondary & & & & & & & \\
\hline VOTB & 0.83 & 0.79 & 2 & 1 & 0.91 & 0.83 & 0.83 \\
\hline HOM65 & 0.65 & 0.65 & 0 & 3 & 0.72 & 0.65 & NA \\
\hline HOM79 & 0.79 & 0.79 & 0 & 3 & 0.89 & 0.79 & NA \\
\hline
\end{tabular}

In the three secondary treatments, VOTB, HOM65, and HOM79 there also exist both All Vote and SVC. In treatment VOTB there exist two SVC equilibria and in each of HOM65 and HOM79 there exist three. Thus voters face strategic uncertainty in all five treatments. However, in the treatments in which there are multiple SVC equilibria, the SVC equilibria involve weakly dominated strategies and we might expect that the All Vote equilibria are more likely to be focal and thus more likely to be observed than in both the ABS and VOT treatments. 
Hence, we expect the participation rates of all voters in HOM65 and HOM79 to be equivalent to the participation rates of voters with high quality information in the ABS, VOT, and VOTB treatments.

In contrast, if voters with low quality information are instead following a norm of "letting the experts decide" we would expect that in ABS, VOT, and VOTB, we would observe voters with low quality information abstaining rather than participating. In ABS and VOT, such behavior will be the same as in the SVC equilibrium, but in VOTB, the behavior will be different from the SVC equilibrium predictions.

\section{Experimental Analysis}

\section{Procedures}

The experiment was conducted at the Laboratory for Experimental Economics at the University of Copenhagen. ${ }^{4}$ The experiment was conducted entirely via computers and programmed in z-Tree [Fischbacher (2007)]. Communication between subjects outside of the computer interface was not allowed. After the experimenter went over the instructions, the subjects answered a set of control questions to verify their understanding of the experiment. The instructions for the experiment are in the Appendix.

In the beginning of the experiment the subjects were randomly divided into groups of three and remained in the same groups throughout the experiment, a fixed matching procedure. The groupings were anonymous, that is, the subjects did not know which of the other subjects were in their groups. The use of repeated interaction is desirable for two reasons: 1) the types of voting situations we focus on-voting in legislatures and committees-are often instances where the participants repeatedly interact and, as we have designed the experiment, know the overall quality of other voters' information and 2) experimental research on coordination games has demonstrated that fixed matching procedures facilitate coordination of subjects on Pareto

\footnotetext{
${ }^{4}$ We used ORSEE to recruit subjects, see Greiner (2004).
} 
Optimal equilibria..$^{5}$

A period in the experiment progressed as follows. Subjects could see two boxes on their computer monitors, a red and a blue box. One of the boxes was randomly chosen to hold a prize. The box chosen was the same for all groups in each period, but randomized across periods. Subjects were only told that the prize was with equal probability in one of the boxes, but not which box. Each subject was given a private signal, either red or blue, about which box might hold the prize. The quality of the signals depended upon a voter's type and were fixed at the values in Table 1 above. In treatments where the signal qualities varied-ABS, VOT, and VOTB-which subjects were designated to receive a high quality signal and which were designated to receive a low quality signal was randomly chosen in each period. Subjects knew the quality of their own signal and the qualities of the two other group members' signals, but only the content of their own signal. After receiving their signals, subjects chose whether to vote for red, blue, or abstain. If the majority voted correctly, the subjects were given a payoff of 30 points and if the majority voted incorrectly they were given -70 points. Ties were broken by a random draw. Subjects' earnings across periods were cumulated during the experiment and at the end of the experiment the total points earned by subjects were converted to Danish Kroner (DKK) at a rate of 6 points per DKK.

\section{Sequences of Treatment}

We conducted nine sessions for a total of 141 subjects. We ordered the treatments in four different sequences: ABS-VOT, VOT-ABS, HOM65-ABS, VOT-VOTB-HOM79, as summarized in Table 2 below. We used both a within and between subjects design.

\footnotetext{
${ }^{5}$ See Clark and Sefton (2001). Devetag and Ortmann (2007) review the literature. Ali, Goeree, Kartik, and Palfrey (2008) experimentally compare ad hoc and standing committee voting without abstention and find that the results are largely consistent.
} 


\begin{tabular}{|c|c|c|c|c|c|}
\hline \multicolumn{5}{|c|}{ Table 2: Treatment Sessions and Sequences } \\
\hline & & \multicolumn{3}{|c|}{ Periods } & No. of \\
\hline Sessions & Sequence & $1-30$ & $31-60$ & & Subjects \\
\hline 1 & ABS-VOT & ABS & VOT & & 24 \\
\hline 2 & VOT-ABS & VOT & ABS & & 21 \\
\hline 3 & ABS-VOT & ABS & VOT & & 12 \\
\hline 4 & VOT-ABS & VOT & ABS & & 15 \\
\hline 5 & HOM65-ABS & HOM65 & ABS & & 9 \\
\hline 6 & HOM65-ABS & HOM65 & ABS & & 18 \\
\hline & & $1-20$ & $21-40$ & $41-60$ & \\
\hline 7 & VOT-VOTB-HOM79 & VOT & VOTB & HOM79 & 9 \\
\hline 8 & VOT-VOTB-HOM79 & VOT & VOTB & HOM79 & 6 \\
\hline 9 & VOT-VOTB-HOM79 & VOT & VOTB & HOM79 & 27 \\
\hline
\end{tabular}

In particular, we used a within subjects design to compare ABS and VOT, holding subjects and groups constant to compare the effects on subjects' choices of two treatments with asymmetric information in one treatment subjects are expected to choose as in the SVC equilibrium and the other subjects are expected to choose as in the All Vote equilibrium. We also used a between subjects design to compare the effects of the sequence on these two treatments, using two sequences ABS-VOT and VOT-ABS. As noted above, these are our two principal treatments and are designed purposely so that the benefits from coordination on the predicted Pareto Optimal equilibrium in each case is equivalent.

We used the sequences HOM65-ABS as well to compare with the sequence VOT-ABS. That is, in both VOT and HOM65 we expect that subjects will coordinate on the All Vote equilibria. However, the coordination in HOM65 is arguably more focal than in VOT, since in HOM65 the SVC equilibria are multiple and involve using weakly dominated strategies. This may affect the ability of voters to coordinate in the ABS treatment. That is, there may be some greater tendency to coordinate on the All Vote equilibrium in the ABS treatment when it is preceded by the HOM65 than when it is preceded by the VOT treatment. Our experimental design allows us to evaluate whether such spillover effects occur. Finally, we used a within subjects design to compare the effects of the three treatments of VOT, VOTB, HOM79, holding subjects and groups constant to consider the effects on subjects' choices of three different treatments where all voters are always predicted to participate. 


\section{Observed Individual Behavior}

\section{Aggregate Individual Choices}

Table 3 summarizes the aggregate vote choices of subjects in the five treatments. First, we find that $96.47 \%$ of the subjects who participated in the elections voted their signals. This suggests that the subjects largely understood the experimental procedures. Second, as predicted, we find only slight evidence of differences in voting behavior between the HOM65 and HOM79 treatments $\left[\chi^{2}\right.$ statistic $\left.=4.54, \operatorname{Pr}=0.10\right] . \quad$ Even though the quality of information is less in HOM65 than in HOM79, in both treatments voters participated in large percentages, 89.51\% and $88.57 \%$, respectively.

\begin{tabular}{|c|c|c|c|c|c|}
\hline \multicolumn{6}{|c|}{ Table 3: Aggregate Individual Behavior } \\
\hline & & \multicolumn{3}{|c|}{ Percentage Vote Choices } & \\
\hline Treatment & Voter Type & Not Signal & Abstain & Signal & Obs. \\
\hline ABS & $p=0.90$ & 1.01 & 0.51 & 98.48 & 990 \\
\hline & $q=0.65$ & 0.56 & 91.72 & 7.73 & 1,980 \\
\hline VOT & $p=0.83$ & 0.61 & 0.71 & 98.67 & 980 \\
\hline & $q=0.79$ & 2.87 & 41.58 & 55.54 & 2,020 \\
\hline VOTB & $p=0.83$ & 3.21 & 1.96 & 94.82 & 560 \\
\hline & $q=0.79$ & 7.14 & 31.79 & 61.07 & 280 \\
\hline HOM65 & $p=q=0.65$ & 3.46 & 7.04 & 89.51 & 810 \\
\hline HOM79 & $p=q=0.79$ & 5.48 & 5.95 & 88.57 & 840 \\
\hline
\end{tabular}

Third, we find significant differences between the vote choices by treatment. Some of these differences are as expected. We find that voters with high quality information participated at high rates in both the ABS and VOT treatments, $98.48 \%$ and $98.67 \%$, respectively. ${ }^{6}$ We also find that in the ABS treatment voters with low quality information abstain a vast majority of the time as predicted, $91.72 \%$, and they participated a majority of the time in the VOT and VOTB treatments, voting their signals $55.54 \%$ and $61.07 \%$ of the time, respectively. Thus, as expected, there is a significant difference in the behavior of voters with low quality information between the ABS and both VOT and VOTB treatments $\left[\chi^{2}\right.$ statistics $=1100(\mathrm{Pr}=0.00)$ and $669.05(\mathrm{Pr}=0.00)$, respectively]. We can conclude that when the information asymmetry is reduced, voters with low quality information are more likely to participate.

\footnotetext{
${ }^{6}$ As expected, the difference between in voting behavior of voters with high quality information in the two treatments is not statistically significant $\left[\chi^{2}\right.$ statistic $\left.=1.32, \operatorname{Pr}=0.52\right]$.
} 
The above differences are as predicted. But we surprisingly find other significant differences that are not expected by standard theory. Specifically, although voters with low quality information are participating in the VOT and VOTB treatments a majority of the time, unexpectedly their voting behavior is significantly different from voters with high quality information in those treatments and all voters in the HOM65 and HOM79 treatments, which is not expected. ${ }^{7}$ Furthermore, voting behavior is also significantly different among these voters in the VOT treatment than in the VOTB treatment $\left[\chi^{2}\right.$ statistic $\left.=20.41, \operatorname{Pr}=0.00\right]$. Thus, voters with low quality information appear to abstain more when information asymmetry exists and also abstain more when in greater numbers.

It is important to remember that the VOTB and HOM79 treatments were conducted using a within subject design, so the differences between these treatments are estimated using the same subjects and controlling for subject specific unobservables. However, the differences between VOT and VOTB may also reflect some learning by subjects to coordinate on the All Vote equilibrium since the VOTB treatments follows a VOT treatment. We can be more confident of the differences between VOTB and HOM79 since we find there is no significant difference in the behavior of subjects between HOM65 (which used different subjects and was not preceded by another treatment) and HOM79.

Along with the surprising results with respect to voters with low quality information, we find unexpected significant differences in the behavior of voters with high quality information across treatments. The voters with high quality information in the ABS, VOT, and VOTB treatments participate significantly more than the voters in the HOM65 and HOM79 treatments. ${ }^{8}$ Thus,

\footnotetext{
${ }^{7}$ The $\chi^{2}$ statistics comparing behavior of voters with low quality information to those with high quality information in treatments VOT and VOTB are $582.45(\mathrm{Pr}=0.00)$ and $171.26(\mathrm{Pr}=0.00)$, respectively. The $\chi^{2}$ statistics comparing behavior of voters with low quality information in VOT with all voters in HOM65 and HOM79 are $320.53(\mathrm{Pr}=0.00)$ and $352.31(\mathrm{Pr}=0.00)$, respectively. The $\chi^{2}$ statistics comparing behavior of voters with low quality information in VOTB with all voters in HOM65 and HOM79 are $122.03(\mathrm{Pr}=0.00)$ and $133.35(\operatorname{Pr}=0.00)$, respectively.

${ }^{8}$ The $\chi^{2}$ statistics comparing behavior of voters with high quality information in the ABS treatment with HOM65 and HOM79 are $71.62(\mathrm{Pr}=0.00)$ and $79.24(\mathrm{Pr}=0.00)$, respectively. The $\chi^{2}$ statistics comparing behavior of voters with high quality information in the VOT treatment with HOM65 and HOM79 are 72.42 (Pr $=0.00)$ and $81.99(\operatorname{Pr}=0.00)$, respectively. The $\chi^{2}$ statistics comparing behavior of voters with high quality information in the VOTB treatment with HOM65 and HOM79 are $18.24(\mathrm{Pr}=0.00)$ and $17.47(\mathrm{Pr}=0.00)$,
} 
the information asymmetry appears to also affect those voters with high quality information, causing them to participate at significantly higher rates than they would if information quality is homogenous. Furthermore, we find that voters with high quality information abstain significantly more in the VOTB treatment, when there are two such voters, than in the ABS and VOT treatments $\left[\chi^{2}\right.$ statistics $=17.49(\mathrm{Pr}=0.00)$ and $20.79(\mathrm{Pr}=0.00)$ respectively $]$. Abstention appears higher when more than one voter has the same information quality, regardless of whether the voter has high quality or low quality information or even if the information quality is homogeneous across voters.

Since our estimates involve some repeated observations of subjects' choices, we also estimate multinomial probits of voter choices clustered by subject which are presented in Table 4 below. In the estimation reported on in the first half of the table pools data from voters with high quality information across treatments with all voters in the HOM65 and HOM79 treatments, while the estimation reported on in the second half pools data from voters with low quality information across treatments with all voters in the HOM65 and HOM79 treatments. We find that the comparisons made above hold controlling for the repeated observations.

respectively. 


\begin{tabular}{|c|c|c|c|c|}
\hline \multicolumn{5}{|c|}{ ABS is omitted category } \\
\hline & \multicolumn{4}{|c|}{ Vote Choice (High Voters Pooled with HOM Voters) } \\
\hline & \multicolumn{2}{|c|}{ Not Signal } & \multicolumn{2}{|c|}{ Abstain } \\
\hline Indep. Var. & Coef. & Robust Std. Err. & Coef. & Robust Std. Err. \\
\hline VOT & -0.25 & 0.29 & 0.16 & 0.40 \\
\hline VOTB & $0.69^{*}$ & 0.38 & $0.76^{* *}$ & 0.34 \\
\hline HOM65 & $0.83^{* *}$ & 0.33 & $1.57^{* * *}$ & 0.36 \\
\hline HOM79 & $1.11^{* * *}$ & 0.31 & $1.50^{* * *}$ & 0.32 \\
\hline Constant & $-3.26^{* * *}$ & 0.23 & $-3.59 * * *$ & 0.25 \\
\hline \multicolumn{5}{|c|}{ Wald $\chi^{2}=59.64$, Log. Like. $=-989.55$, Obs. $=4180$, Clusters $=141$} \\
\hline & \multicolumn{4}{|c|}{ Vote Choice (Low Voters Pooled with HOM Voters) } \\
\hline & \multicolumn{2}{|c|}{ Not Signal } & \multicolumn{2}{|c|}{ Abstain } \\
\hline Indep. Var. & Coef. & Robust Std. Err. & Coef. & Robust Std. Err. \\
\hline VOT & $-0.62^{* *}$ & 0.27 & $-2.25^{* * *}$ & 0.22 \\
\hline VOTB & -0.19 & 0.31 & $-2.56^{* * *}$ & 0.30 \\
\hline HOM65 & $-1.05^{* * *}$ & 0.34 & $-4.02^{* * *}$ & 0.28 \\
\hline HOM79 & $-0.77^{* * *}$ & 0.30 & $-4.11^{* * *}$ & 0.27 \\
\hline Constant & $-1.39 * * *$ & 0.22 & $2.00^{* * *}$ & 0.18 \\
\hline \multirow{2}{*}{\multicolumn{5}{|c|}{ Wald $\chi^{2}=352.39$, Log. Like. $=-3138.54$, Obs. $=5930$, Clusters $=141$}} \\
\hline & & & & at $1 \%$ level \\
\hline
\end{tabular}

\section{Patterns of Individual Choices}

The analysis above examines voter choices in the aggregate comparing choices between and within subjects. But our within-subjects design allows us to analyze the pattern of choices made by a single subject for different treatments. Table 5 presents a summary of the patterns of behavior of subjects by combinations of treatments. We classify a subject as mostly choosing a strategy in a particular treatment if that subject used that strategy more than $50 \%$ of the time. The strategy combinations that fit either an SVC equilibrium or the All Vote equilibrium are highlighted in bold in the table. As the table shows, 71 out of 72 of the subjects in the treatment combinations of ABS and VOT mostly chose strategies across treatments that fit one of the equilibria. The table also illustrates that exactly half of the subjects in this combination mostly chose strategies in ABS that coincided with the SVC equilibrium and mostly chose strategies in VOT that coincided with the All Vote equilibrium, but nearly half, over 47\%, mostly chose strategies that coincided with the SVC equilibrium in both ABS and VOT. 
Table 5: Patterns of Individual Choices by Treatment Combination (Equilibrium in Bold) LA $=$ mostly abstain low voter, LS = mostly vote signal low voter, \& LNS = mostly not vote signal low voter, HA, HS, \& HNS similarly defined.

A, S, and NS similarly defined for hom. infor. case. Missing Cases Unobserved. Combinations

\begin{tabular}{|c|c|c|c|c|c|c|c|c|c}
\hline \multicolumn{2}{|c|}{ ABS \& VOT (72 Obs.) } & \multicolumn{3}{c|}{ ABS \& HOM65 (27 Obs.) } & \multicolumn{4}{c|}{ VOT, VOTB, \& HOM79 (42 Obs.) } \\
\hline ABS & VOT & Per. & ABS & HOM & Per. & VOT & VOTB & HOM & Per. \\
\hline LA HS & LA HS & $\mathbf{4 7 . 2 2}$ & LA HS & S & $\mathbf{8 1 . 4 8}$ & LNS HA & LS HS & S & 2.38 \\
\hline LA HS & LS HS & $\mathbf{5 0 . 0 0}$ & LA HS & NS & 3.70 & LA HS & LA HS & S & $\mathbf{1 1 . 9 0}$ \\
\hline LA HS & LNS HS & 1.39 & LS HS & S & $\mathbf{1 4 . 8 1}$ & LA HS & LA HS & NS & 2.38 \\
\hline LS HS & LS HS & $\mathbf{1 . 3 9}$ & & & & LA HS & LS HS & S & $\mathbf{1 9 . 0 5}$ \\
\hline & & & & & & LS HS & LA HS & S & $\mathbf{1 6 . 6 7}$ \\
\hline & & & & & & LS HS & LS HS & S & $\mathbf{3 3 . 3 3}$ \\
\hline & & & & & & LS HS & LNS HS & S & 2.38 \\
\hline & & & & & & LNS HS & LS HA & S & 2.38 \\
\hline & & & & & & LNS HS & LS HS & S & 7.14 \\
\hline & & & & & & LNS HS & LNS HS & NS & 2.38 \\
\hline
\end{tabular}

For the treatment combination of ABS and HOM65, we find similarly that 26 out of the 27 subjects mostly chose strategies across treatments that fit one of the equilibria. A large majority, over $81 \%$, mostly chose strategies that coincided with the SVC equilibrium in ABS and the All Vote equilibrium in HOM65, while nearly 15\% mostly chose strategies that coincided with the All Vote equilibrium in both treatments. This difference between the combinations ABS and HOM65 and ABS and VOT suggest that there is a spillover effect from the HOM65 treatment on behavior in the ABS treatment given that ABS was the second treatment in the sequence using that combination. We investigate this further after we discuss group behavior.

We observe the most cases where subjects mostly chose strategies that do not fit equilibria in the treatment combination of VOT, VOTB, and HOM79, with 7 out of 42 subjects in that category. Five of the seven mostly chose strategies involving voting contrary to their signals in either VOT or VOTB, but did vote their signals in HOM79, which was the last treatment experienced, which suggests that in these cases the subjects were making errors in the earlier treatments. Of the 35 subjects who mostly chose equilibrium strategies in this treatment combination, we find that nearly $12 \%$ mostly chose strategies consistent with the SVC equilibrium in VOT and VOTB, and the All Vote equilibrium in HOM79; $19 \%$ mostly chose strategies consistent with 
the SVC equilibrium in VOT, and the All Vote equilibrium in VOTB and HOM79; and one third mostly chose strategies consistent with the All Vote equilibrium in all three treatments, which was the expected or predicted choice. Of note is the fact that we find little evidence that subjects with high quality information in the VOTB abstained at the rate predicted by the SVC equilibrium in this treatment since all but one of the 42 subjects mostly chose to vote their signal when they had high quality information in this treatment and the SVC equilibrium would predict that at least half of the time subjects would choose to abstain.

\section{Summary of Observed Individual Behavior}

In summary, we find that:

1. Mostly, there is little difference in behavior between voters with high quality information across treatments with asymmetric information, but these voters participate at a higher rate than voters in treatments with homogeneous information and participate a little less when there is more than one vote with high quality information.

2. Voters with low quality information are most likely to abstain in the ABS treatment, but they abstain more in the VOT treatment than the VOTB treatment, and abstain more in the VOTB treatment than in the treatments with homogeneous information.

3. It appears that voters in the ABS treatment are coordinating on the SVC equilibrium, as expected, and that when the quality of information is homogeneous voters appear to coordinate on the All Vote equilibrium, as expected.

4. But the results also suggest heterogeneity in the individual behavior in the VOT and VOTB treatments and that some voters may be coordinating on the All Vote equilibria while others may be coordinating on a SVC equilibrium in both cases. Furthermore the low abstention rate of voters with high quality information with the sizeable abstention of voters with low quality information in the VOTB treatment suggests that they may be coordinating on a nonequilibrium choice and the observations may simply reflect behavior that does not fit with either the SVC or All Vote equilibrium predictions at the group level. 
To determine if differences in how voters coordinate explain this last result, we turn to an analysis of group behavior.

\section{Observed Group Behavior Aggregate Group Choices}

Table 6 summarizes aggregate group behavior according to whether a group's choices fit the predicted behavior in an SVC equilibrium, an All Vote equilibrium, or fit neither. Specifically, in all treatments a group's choice was classified as fitting an All Vote equilibrium if all group members voted their signals. In the treatments ABS and VOT a group's choice was classified as fitting an SVC equilibrium if the voter with high quality information voted his or her signal and the two voters with low quality information abstained; in the treatment VOTB a group's choice was classified as fitting an SVC equilibrium if one of the voters with high quality information voted his or her signal and the other two voters abstained; and in the treatments HOM65 and HOM79 a group's choice was classified as fitting an SVC equilibrium if only one voter participated and voted his or her signal.

\begin{tabular}{|c|c|c|c|c|}
\hline \multicolumn{5}{|c|}{ Table 6: } \\
\hline & \multicolumn{5}{|c|}{ Percentage of Groups } \\
\hline Treatment & Other & SVC Equil. & All Vote Equil. & Obs. \\
\hline ABS & 13.54 & 84.65 & 1.82 & 990 \\
\hline VOT & 29.50 & 29.60 & 40.90 & 1,000 \\
\hline VOTB & 44.64 & 1.07 & 54.29 & 280 \\
\hline HOM65 & 26.30 & 1.85 & 71.85 & 270 \\
\hline HOM79 & 28.21 & 1.07 & 70.71 & 280 \\
\hline
\end{tabular}

As expected, large majorities of groups coordinate on the SVC equilibrium in the ABS treatment and on the All Vote equilibrium in the HOM65 and HOM79 with very few observations of the other equilibria observed. There is no significant difference in the ability of groups to coordinate on the All Vote equilibria in the HOM65 and HOM79 treatments $\left[\chi^{2}\right.$ statistic $=0.79$, $\operatorname{Pr}=0.68]$, so there is no evidence that the difference in information quality across treatments affects the behavior of the groups in these treatments where all subjects' information quality is equal. 
In the VOT treatment over $40 \%$ of the groups coordinate on the All Vote equilibrium as well. However, this is significantly less than in the VOTB, HOM65, and HOM79 treatments $\left[\chi^{2}\right.$ statistics $=100.45,112.72$, and 117.34, respectively, all $\mathrm{Pr}=0.00] . \quad$ This is partly explained by the fact that nearly $30 \%$ of the groups in the VOT treatment chose according to the SVC equilibrium. Thus, the tendency of voters with low quality information to abstain in the VOT treatment does appear to reflect voters coordinating on an SVC equilibrium. However, nearly $30 \%$ of the time the groups fail to coordinate on either the SVC or the All Vote equilibria in this treatment as well. Failure to coordinate in VOT is significantly higher than in the ABS treatment $\left[\chi^{2}\right.$ statistic $\left.=74.98, \operatorname{Pr}=0.00\right]$. Interestingly, coordination is also a problem for groups in the HOM65 and HOM79 treatments. Failure to coordinate in the HOM65 and HOM79 treatments is also significantly higher than in the ABS treatment $\left[\chi^{2}\right.$ statistics $=25.36$ and 33.69, respectively, both $\operatorname{Pr}=0.00]$ but not significantly different from the VOT treatment $\left[\chi^{2}\right.$ statistics $=1.06(\operatorname{Pr}=0.30)$ and $0.17(\operatorname{Pr}=0.68)$, respectively $]$.

Coordination appeared the most difficult for subjects in the VOTB treatment, with almost $45 \%$ of groups not coordinating on either an All Vote or SVC equilibrium. The incidence of coordination failure is significantly higher in the VOTB treatment than in all of the other treatments. ${ }^{9}$ Yet, unlike the VOT treatment, there is little evidence that groups were attracted to the SVC equilibrium as we observe only 3 group choices consistent with that equilibrium, the same as in HOM79. In fact the percentage of choices consistent with the SVC equilibrium in VOTB is not significantly different from the choices in HOM65 either $\left[\chi^{2}\right.$ statistic $=0.58(\operatorname{Pr}$ $=0.45)]$. As in our analysis of aggregate individual behavior, we have repeated observations of groups' choices. Therefore we also estimate a multinomial probit equation of group choices which is reported on in Table 7 .

\footnotetext{
${ }^{9}$ The $\chi^{2}$ statistic for the comparison with the ABS treatment is 130.10 , with the VOT treatment is 22.75 , with the HOM65 treatment is 20.17 , and with the HOM79 treatment is 16.32 , all with $\operatorname{Pr}=0.00$.
} 


\begin{tabular}{|c|c|c|c|c|}
\hline \multirow{2}{*}{\multicolumn{5}{|c|}{$\begin{array}{c}\text { Table 7: Multinomial Probit of Group Choi } \\
\text { ABS Treatment is the Omitted Category }\end{array}$}} \\
\hline & & & & \\
\hline & \multicolumn{4}{|c|}{ Equilibrium Choice } \\
\hline & \multicolumn{2}{|c|}{ Not SVC and Not All Vote } & \multicolumn{2}{|r|}{ All Vote } \\
\hline Indep. Var. & Coef. & Robust Std. Err. & Coef. & Robust Std. Err. \\
\hline VOT & $1.52^{* * *}$ & 0.27 & $2.95^{* * *}$ & 0.46 \\
\hline VOTB & $3.83^{* * *}$ & 0.29 & $5.17^{* * *}$ & 0.53 \\
\hline HOM65 & $3.15^{* * *}$ & 0.24 & $5.18^{* * *}$ & 0.54 \\
\hline HOM79 & $3.46^{* * *}$ & 0.40 & $5.43^{* * *}$ & 0.53 \\
\hline Constant & $-1.53^{* * *}$ & 0.20 & $-2.69 * * *$ & 0.39 \\
\hline \multicolumn{5}{|c|}{ Wald $\chi^{2}=353.16$, Log. Like. $=-2134.31$, Obs. $=2820$, Clusters $=47$} \\
\hline
\end{tabular}

\section{Patterns of Group Choices}

As with individual choices, our within-subjects design and our use of fixed matching procedures allows us to compare group choices across treatments in treatment combinations, holding constant unobservable characteristics about the groups. Table 8 presents an analysis of the patterns of group choices by treatment combinations. We classify a group as mostly coordinating on a given equilibrium over time in a treatment if their choices as a group were consistent with that equilibrium more than $50 \%$ of the time within that treatment. A group is classified as not coordinating on a given equilibrium over time within that treatment otherwise.

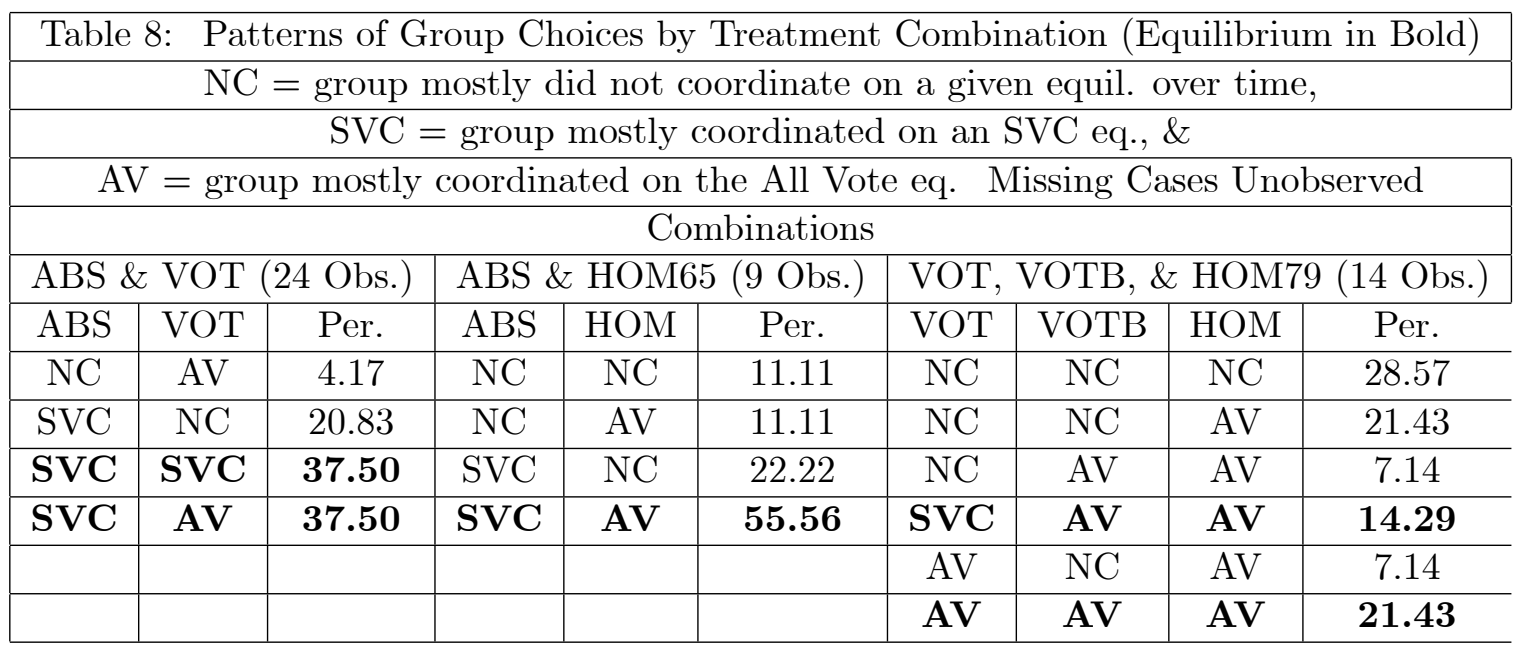

We find that in the treatment combination of ABS and VOT, three quarters of groups were mostly able to coordinate over time in both treatments. They coordinated on the SVC equilibrium in the ABS treatment and half coordinated on the SVC while the other half coordinated 
on the All Vote equilibrium in the VOT treatment, which fits with the individual behavioral patterns discussed above. Five of the six groups who could not coordinate over time in general, were able to coordinate mostly on the SVC equilibrium in the ABS treatment, and the remaining group that could not coordinate over time in general, was able to coordinate mostly on the All Vote equilibrium in the VOT treatment. Thus, no group in this treatment combination did not mostly coordinate over time within at least one of the treatments.

We find that in the treatment combination of ABS and HOM65 there is much less overall coordination over time, with only a little over $55 \%$ of the groups able to mostly coordinate over time on the same equilibrium in the ABS treatment (the SVC equilibrium) and in the HOM65 treatment (the All Vote equilibrium). However, three of the four groups who could not coordinate over time in general, were able to mostly coordinate over time in one of the treatments; two groups coordinated on the SVC equilibrium over time in the ABS treatment even though they did not coordinate over time in the HOM65 treatment, and one of the groups coordinated on the All Vote equilibrium over time in the HOM65 treatment even though they did not coordinate over time in the ABS treatment. Thus, only one group in this treatment combination did not mostly coordinate over time within at least one of the treatments.

We find the least overall coordination over time in the treatment combination VOT, VOTB, and HOM79, with about $36 \%$ of groups mostly coordinating on an equilibrium within a treatment; with a little over $14 \%$ coordinated on the SVC equilibrium in the VOT treatment and the All Vote equilibrium in the VOTB and HOM79 treatments and a little over $21 \%$ coordinate on the All Vote equilibrium in all three treatments. As with the other treatment combinations, most of the groups who did not coordinate overall, did mostly coordinate on an equilibrium in one or two of the treatments. However, four groups did not mostly coordinate on an equilibrium in any of the treatments. 


\section{Analysis of Coordination Failures}

In order to explore the determinants of the coordination failures, we examine the distribution of choices made when groups did not coordinate in Table 9. We find that in the ABS and VOT treatments, a large majority of coordination failures, approximately $81 \%$ and $75 \%$, respectively, occur when the voter with the high quality information votes his or her signal, one voter with low quality information also votes his or her signal, and the second voter with low quality information abstains. These errors then are consistent with voters having trouble coordinating between the SVC and All Vote equilibria. Also somewhat consistent with voters having trouble coordinating between the SVC and All Vote equilibria are the coordination failures in HOM65 where the majority of the coordination failures occurred when only two voters voted their signal and the other voter abstained (63\%). However, in HOM79 the majority of the coordination failures occurred when one or more voters voted contrary to their signals $(53.16 \%)$.

\begin{tabular}{|c|c|c|c|c|c|}
\hline Table 9: Percentage Breakdown of Choices When Coordination Fails \\
\hline \multicolumn{5}{|c|}{ Missing Values Unobserved. } \\
\hline Type of Behavior & \multicolumn{5}{c|}{ Treatment } \\
\hline Type of Behavior & ABS & VOT & VOTB & HOM65 & HOM79 \\
\hline All abstain & 2.99 & 0.34 & & & \\
\hline H \& one L votes signal, other L abstains & 80.60 & 75.25 & & & \\
\hline One L votes signal, H \& other L abstain & 0.75 & 0.68 & & & \\
\hline Two L vote signals \& H abstains & & 3.73 & & & \\
\hline Two H vote signal, L abstains & & & 68.00 & & \\
\hline One H \& L votes signal, other H abstains & & & 5.60 & & \\
\hline Two voters vote signal, other abstains & & & & 63.38 & 46.84 \\
\hline One or more vote against signal & 15.67 & 20.00 & 26.40 & 36.62 & 53.16 \\
\hline Observations & 134 & 295 & 125 & 71 & 79 \\
\hline H = voter with high quality information, L = voter with low quality information \\
\hline
\end{tabular}

A majority of the VOTB coordination failures, $68 \%$, occur when both voters with high quality information vote their signals but the voter with low quality information abstains. This may seem to also be explained by subjects having trouble coordinating. However, we observe only about $6 \%$ of groups failing to coordinate when the voter with low quality information votes his or her signal, one voter with high quality information also votes his or her signal, and the second voter with high quality information abstains, which one would expect to occur if some 
subjects were coordinating on an SVC equilibrium. Given that we also observe very few SVC equilibria in VOTB as evidenced above, the errors in VOTB do not appear to occur because of difficulty in coordinating on a given equilibrium, but to reflect a subject preference for group choices in which the two voters with high quality information participate and the voter with the low quality information abstains, which is not an equilibrium.

\section{Efficiency of Group Choices}

Given that coordination failures occurred and varied by treatment, it is useful to compare the informational efficiency of group choices with the equilibrium predictions. We do so in Table 10 below. We coded a group's choice as a 1 if the group voted for the correct box, 0.5 if the vote was a tie, and 0 if the group voted for the incorrect box. We find that the patterns of observed efficiencies followed the theoretical predictions but the only significant differences by treatment are between the efficiency of the HOM65 treatment and all the other treatments. ${ }^{10}$ The efficiency results also reflect the coordination problems facing the groups as discussed above. We calculated an Efficiency Ratio equal to the Observed Efficiency divided by the Efficiency in the Pareto Optimal equilibrium that measures the relative efficiency of the choices of groups by treatment.

\begin{tabular}{|c|c|c|cc}
\hline \multicolumn{5}{|c}{ Table 10: Informational Efficiency } \\
\hline Treatment & Obs. Eff. & All Vote Eq. Eff. & SVC Eq. Eff. & Eff. Ratio \\
\hline ABS & 0.87 & 0.83 & 0.90 & 0.97 \\
\hline VOT & 0.84 & 0.90 & 0.83 & 0.93 \\
\hline VOTB & 0.86 & 0.91 & 0.83 & 0.95 \\
\hline HOM65 & 0.69 & 0.72 & 0.65 & 0.96 \\
\hline HOM79 & 0.83 & 0.89 & 0.79 & 0.93 \\
\hline
\end{tabular}

\section{Summary of Observed Group Behavior}

In conclusion, we find the following:

1. Groups in the ABS treatment find it easiest to coordinate and largely coordinate on the SVC equilibrium.

\footnotetext{
${ }^{10}$ The $\mathrm{t}$ statistics comparing HOM65 with ABS, VOT, VOTB, and HOM79 are 6.28, 5.35, 5.33, and 4.22, respectively.
} 
2. Groups in the VOT treatment find it less easy to coordinate than in the ABS treatment and vary over whether they coordinate on the SVC equilibrium or the All Vote equilibrium. About half of groups coordinate on the SVC equilibrium and about half coordinate on the All Vote equilibrium.

3. Groups in the VOTB treatment find it most difficult to coordinate and either coordinate on the All Vote equilibrium or the non-equilibrium outcome where the two voters with high quality information participate and the voter with low quality information abstains.

4. Groups in the HOM65 and HOM79 treatments largely coordinated on the All Vote equilibrium as expected, although there are significantly more coordination failures in these treatments than in the ABS treatment.

Thus, we find some evidence that coordination failures occur due to the strategic uncertainty facing voters in the ABS and VOT treatments. We find weaker evidence that coordination failures occur due to strategic uncertainty in the HOM65 and HOM79 treatments. But in the VOTB treatment, we find evidence that suggests that some groups are coordinating on choices that are not an equilibrium.

\section{Sequencing Effects on Coordination}

\section{Individual Behavior}

As noted above, our experimental design allows us to compare the effects of sequencing on voting behavior. Specifically, we conduct sessions where subjects first experienced the ABS treatment and then the VOT treatment (sessions 1 and 3) and vice-versa (sessions 2 and 4). We also conducted two sessions where ABS was preceded by the HOM65 treatment (sessions 5 and 6 ) and three other sessions where VOT preceded other treatments (sessions 7, 8, and 9). As noted we find that subjects are more likely to coordinate on the All Vote equilibrium in the HOM65 treatment than in the VOT treatment. It is useful, then, to compare the VOT-ABS treatments with the HOM65-ABS treatments to determine if more experience with the All Vote equilibria in the HOM65 treatment has a spillover effect on how subjects coordinate in the ABS treatment 
when the SVC equilibrium is Pareto Optimal.

Table 11 summarizes the voting behavior in the ABS and VOT treatments by sequence. First, we find that the overall choices of voters with high quality information in both the VOT and ABS treatments are unaffected by sequence. ${ }^{11}$ However, we find that the choices of voters with low quality information in the VOT and ABS treatments are affected by sequence. First, we find that in the VOT treatment subjects with low quality information who experience the VOT treatment after the ABS treatment are significantly more likely to abstain and vote against their signal less often than when they experience the VOT treatment first. ${ }^{12}$

\begin{tabular}{|c|c|c|c|c|c|c|}
\hline \multicolumn{2}{|c|}{ Table 11: Individual Behavior in ABS and VOT Treatments by Sequence } \\
\hline & & & \multicolumn{2}{c|}{ Percentage Vote Choices } & \\
\hline Treatment & Sequence & Voter Type & Not Signal & Abstain & Signal & Obs. \\
\hline VOT & ABS-VOT & $p=0.83$ & 0.56 & 0 & 99.44 & 360 \\
\hline & & $q=0.79$ & 0.56 & 44.31 & 55.14 & 720 \\
\hline & VOT First Treat. $^{13}$ & $p=0.83$ & 0.65 & 1.13 & 8.23 & 620 \\
\hline & & $q=0.79$ & 4.15 & 40.08 & 55.77 & 1,300 \\
\hline ABS & ABS-VOT & $p=0.90$ & 1.11 & 0.28 & 98.61 & 360 \\
\hline & & $q=0.65$ & 1.25 & 93.06 & 5.69 & 720 \\
\hline & VOT-ABS & $p=0.90$ & 0.83 & 0 & 99.17 & 360 \\
\hline & & $q=0.65$ & 0.14 & 96.94 & 2.92 & 720 \\
\hline & HOM65-ABS & $p=0.90$ & 1.11 & 1.48 & 97.41 & 270 \\
\hline & & $q=0.65$ & 0.19 & 82.96 & 16.85 & 540 \\
\hline
\end{tabular}

Second, we also find that in the ABS treatment subjects with low quality information abstain significantly more, vote their signals and vote against their signals significantly less in the VOTABS treatment than in the ABS-VOT treatment. ${ }^{14}$ Both of these results may reflect the fact that these subjects have more experience and thus have a better understanding of the voting game and that the experience outweighs any spillover effect of first experiencing the previous treatments. Third, however, we find that in the ABS treatment subjects with low quality information vote their signals significantly more and abstain significantly less in the HOM65-

\footnotetext{
${ }^{11}$ The $\chi^{2}$ statistic for the comparison of the ABS treatment across the three sequences it appears for voters with high quality information is 7.51, which is not significant. The statistic for the comparison of the VOT treatment between the sequence ABS-VOT and the results of sequences VOT-ABS and VOT-VOTB-HOM79 is 4.13, which is also not significant.

${ }^{12}$ The $\chi^{2}$ statistic for the comparison is 22.92 .

${ }^{13}$ This includes observations from sequences VOT-ABS and VOT-VOTB-HOM79.

${ }^{14}$ The $\chi^{2}$ statistic for the comparison of the two treatments is 13.43 .
} 
ABS treatment than in either the ABS-VOT or VOT-ABS treatments, which suggests that there is some spillover effect from experience with primarily coordinating on the All Vote equilibria. ${ }^{15}$

Although we find that there are some significant differences in the behavior of subjects with low quality information as the sequence of treatments is varied, importantly, when we control for sequence, our findings of significant differences between the ABS and VOT treatments are robust. That is, when we consider the subjects with low quality information, controlling for sequence, we find significantly more of these subjects voting their signals and abstaining less in the VOT treatment than in the ABS treatment, as we found in the aggregate. ${ }^{16}$ Thus, any experience effects related to sequence are overwhelmed by the treatment effects.

\section{Group Behavior}

The results on the effect of sequence on individual behavior are thus mixed as to whether spillover effects occur. We now turn to an analysis of the effects of sequence on group behavior by examining group behavior in the ABS and VOT treatments by sequence. Table 12 summarizes these results.

\begin{tabular}{|c|c|c|c|c|c|}
\hline \multicolumn{6}{|c|}{ Table 12: Group Behavior in ABS and VOT Treatments by Sequence } \\
\hline & & \multicolumn{3}{|c|}{ Percentage of Groups } & \\
\hline Treatment & Sequence & Other & SVC Equil. & All Vote Equil. & Obs. \\
\hline VOT & ABS-VOT & 26.11 & 31.67 & 42.22 & 360 \\
\hline & VOT First Treat. ${ }^{17}$ & 31.41 & 28.44 & 40.16 & 640 \\
\hline ABS & ABS-VOT & 12.50 & 86.11 & 1.39 & 360 \\
\hline & VOT-ABS & 6.11 & 93.61 & 0.28 & 360 \\
\hline & HOM65-ABS & 24.81 & 70.74 & 4.44 & 270 \\
\hline
\end{tabular}

First, we find that sequence has no significant effect on group behavior in the VOT treatments, so the experience effect for voters with low quality information in the VOT treatments noticed above has only a minor effect group coordination. ${ }^{18}$ However, we do find that sequence does have a significant effect on group coordination in the ABS treatments, which mirrors the effects found above with individual behavior. That is, we find that when we compare the

\footnotetext{
${ }^{15}$ The $\chi^{2}$ statistic for the comparison with ABS-VOT is 44.62 and with VOT-ABS is 74.09.

${ }^{16}$ The $\chi^{2}$ statistic for the comparison in the sequence ABS-VOT is 415.85 and in VOT-ABS is 464.28.

${ }^{17}$ This includes observations from sequences VOT-ABS and VOT-VOTB-HOM79.

${ }^{18}$ The $\chi^{2}$ statistic $=3.24$ for the comparison.
} 
group choices between ABS-VOT and VOT-ABS, groups in the VOT-ABS sequence coordinate on the SVC equilibrium significantly more. ${ }^{19}$ But when the groups first treatment is HOM65 compared to when their first treatment is VOT, the subjects coordinate on the SVC equilibrium significantly less. ${ }^{20}$ Somewhat surprisingly, most of the difference is made up in coordination failures rather than coordination on the All Vote equilibrium. Of these coordination failures a large majority, over $88 \%$, occur because one voter with low quality information is participating while the other is abstaining. Thus, the experience of HOM65 prior to the ABS treatment leads to more coordination failures while the experience of VOT as a first treatment leads to less coordination failures. We suspect that the difference occurs because in VOT some subjects are already coordinating on the SVC equilibria and are familiar with the voting game with asymmetric information.

Again, notably, although we find that sequence can have an effect, the treatment effects of $\mathrm{ABS}$ versus VOT are robust to controlling for sequence. We find that in both sequences ABS-VOT and VOT-ABS, the choices of the groups are significantly different, with groups coordinating on the SVC equilibria significantly more often and the All Vote equilibria significantly less often in the ABS treatment than in the VOT treatment. ${ }^{21}$ Thus, our treatment effects overwhelm the sequencing effects.

\section{Experience within a Treatment}

\section{Individual Behavior}

As we have noted, evidence suggests that in the ABS treatment subjects who have experience make choices more consistent with the theoretical predictions. In this subsection we explore the effects of experience within a treatment on individual behavior. Figure 2 below illustrates how subjects choices change over the periods within a treatment. As the Figure shows there appears to be some evidence of changes in behavior during the ABS treatment. Specifically,

\footnotetext{
${ }^{19}$ The $\chi^{2}$ statistic for the comparison is 11.69 .

${ }^{20}$ The $\chi^{2}$ statistic is 60.82 .

${ }^{21}$ The $\chi^{2}$ statistic is 245.51 for the sequence ABS-VOT and 286.87 for the sequence VOT-ABS.
} 
voters with low quality information in the ABS treatment significantly increase their abstention rates over time, but there is little change over time in the behavior of other voters. In Appendix B we report estimates of separate multinomial logit equations of vote choice by treatment as a linear function of the period in the treatment in each session. ${ }^{22}$ We find some evidence of learning in all almost sessions and treatments.

Figure 2

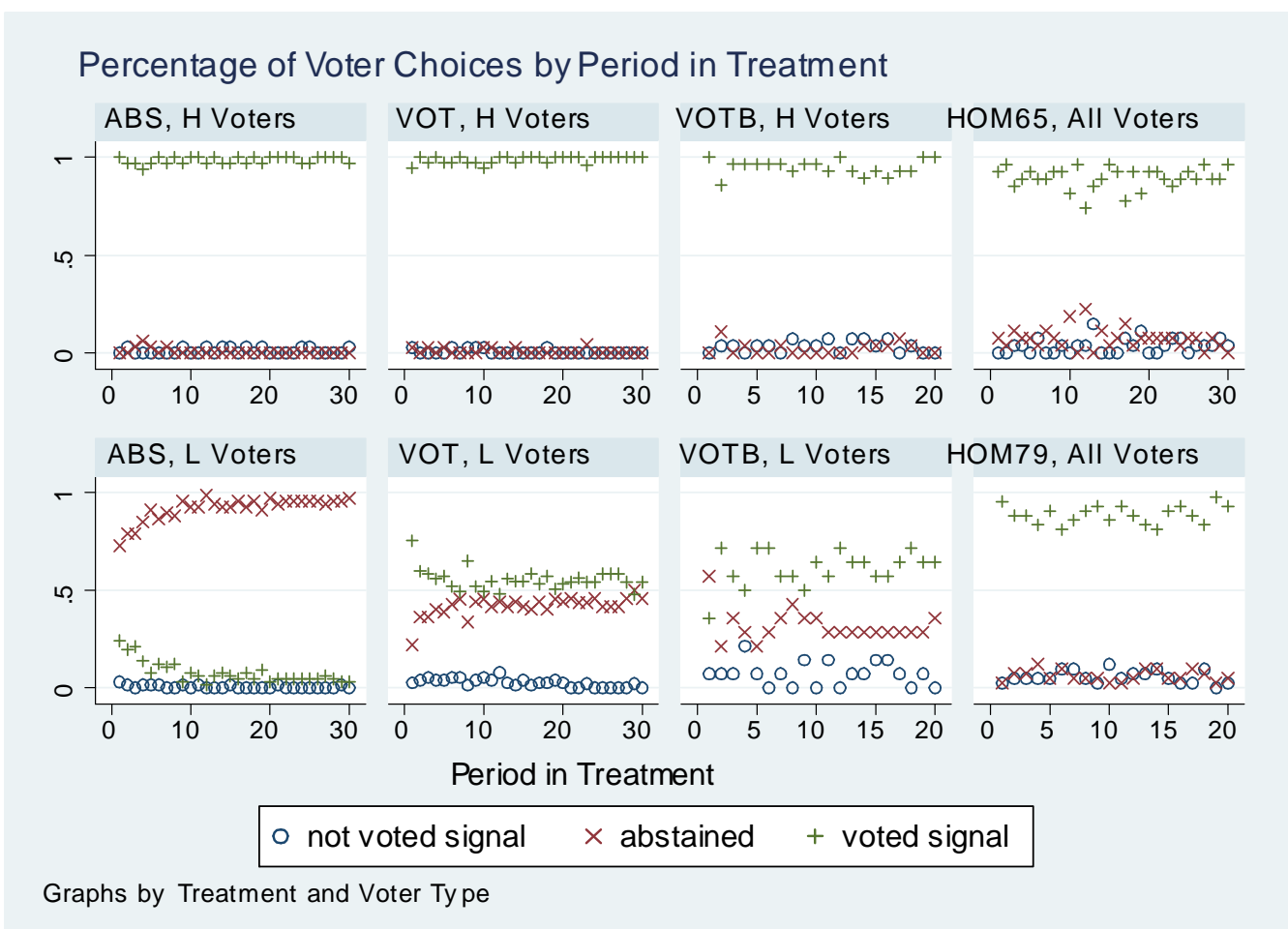

\section{Group Behavior}

We also examine the extent that the behavior of groups change during a treatment. Figure 3 illustrates how group choices change over time during a treatment. As with individual behavior, the graphs show evidence of change over time except for the ABS treatment, where we see significant increase in SVC equilibrium choices during a treatment. ${ }^{23}$

\footnotetext{
${ }^{22}$ Multinomial probit estimations failed to converge. We varied the base outcome in the estimations to facilitate convergence in the multinomial logits.

${ }^{23}$ As with the individual behavior over time, we estimated separate probit equations for each treatment with the SVC equilibrium as the dependent variable and period in treatment interacted with session clustering by group. These results are available from the authors.
} 
Figure 3

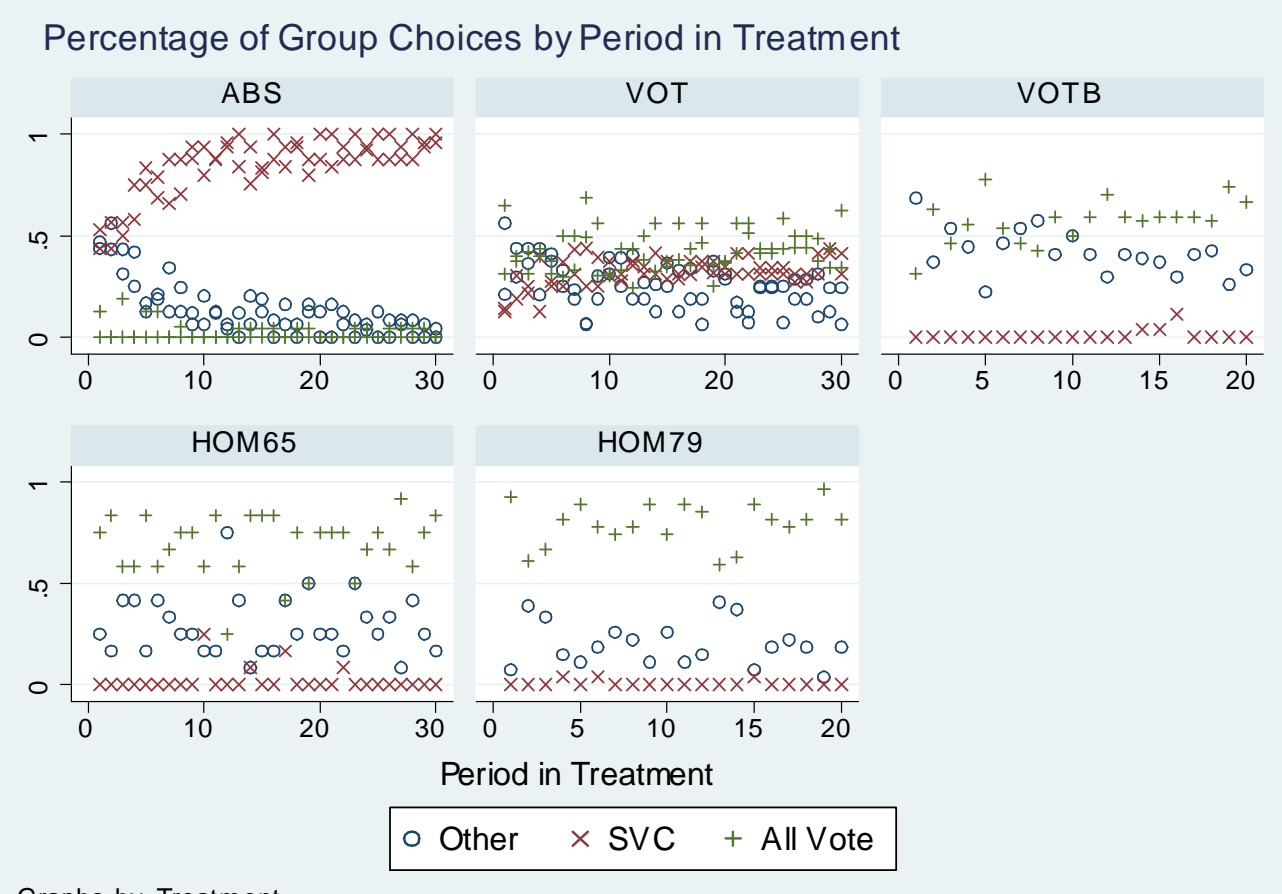

Graphs by Treatment

As with individual choices, we estimated separate multinomial logit equations for group choices by treatment a linear function of the period in the treatment in each session. Tables 14a,b summarizes these estimations. ${ }^{24}$ The standard error was adjusted for the clusters of observations by group. As above, we find some evidence of learning in almost sessions and treatments.

\section{Conclusions}

In this paper we have examined abstention when voters in standing committees are asymmetrically informed and there are multiple pure strategy equilibria-swing voter's curse (SVC) equilibria where voters with low quality information abstain and equilibria when all participants vote their information. When the asymmetry in information quality is large, we find that voting groups largely coordinate on the SVC equilibrium which is also Pareto Optimal. However,

\footnotetext{
${ }^{24}$ Multinomial probit estimations failed to converge. We varied the base outcome in the estimations to facilitate convergence in the multinomial logits.
} 
we find that when the asymmetry in information quality is not large and the Pareto Optimal equilibrium is for all to participate, significant numbers of voters with low quality information abstain. Furthermore, we find that information asymmetry induces voters with low-quality information for groups to coordinate on a non-equilibrium outcome. This suggests that coordination on "letting the experts" decide is a likely voting norm that sometimes validates SVC equilibrium predictions but other times does not. and there are multiple pure strategy Bayesian Nash equilibria-SVC equilibria and equilibria when all participants vote their information. Voters therefore face strategic uncertainty. In contrast to previous studies of the swing voter's curse, in our experiments the quality of subjects' information varies. When the asymmetry in information quality is large, we find that subjects largely coordinate on the SVC equilibrium which is also Pareto Optimal, as predicted. However, we find that when the asymmetry in information quality is not large and the Pareto Optimal equilibrium is for all to participate, significant numbers of voters are attracted to the SVC equilibrium regardless. Furthermore, we find that in one treatment information asymmetry induces voters with low quality information to abstain and for groups to coordinate on a non-equilibrium outcome. When subjects have homogeneous quality of information, we find that they coordinate largely on the All Vote equilibrium as predicted, but their coordination is not as successful as when the quality of information is asymmetric and the asymmetry is large.

Our results suggest that the effects on voters of information asymmetries is significant. Voters with low quality information abstain in response to information asymmetries and voters with high quality information participate more as well. Moreover, the effect appears to influence voters with low quality information to abstain even when doing so is not Pareto Optimal and sometimes not equilibrium behavior as a voting group. Our research suggests that voters with low quality information appear to follow a norm or rule of thumb of "letting the experts decide." Sometimes doing so fits with the equilibrium predictions of the game theoretic model and is Pareto Optimal. But other times we find that indivudals appear to follow the norm 
when it is not Pareto Optimal and, even more striking, when it is not an equilibrium choice.

Our research has implications for understanding the relationship between asymmetries in information quality and turnout in general. That is, we find robust evidence that voters with low quality information are likely to delegate their votes to those with higher quality information and that the delegation can occur even when doing so is suboptimal for the voters and the group as a whole. Because of the suboptimal behavior of voters, appearing to follow the norm or rule of thumb, groups may make more correct choices when information asymmetries are large than when they are small.

\section{Appendix A: Instructions}

Welcome to the experiment. Please do not communicate during the experiment. If you have any questions please raise your hand. You can earn money in this experiment. The amount of money you earn depends on your decisions and the decisions of other participants. All earnings will be paid to you immediately after the experiment. During the experiment, your income will be calculated in points. After the experiment, your income will be converted into Danish kroner (DKK) according to the following exchange rate: 6 points $=1$ DKK. The experiment has 60 periods. All participants are randomly divided into groups of three. The group composition remains constant throughout the experiment. That is, you will be in a group with the same two participants. All participants are anonymous; nobody knows which other participants are in their group, and nobody will be told who was in which group after the experiment.

Each period is structured as follows: (1) A prize is placed in one of two boxes ("red" or "blue"), (2) Each group member receives information about where the prize is hidden, (3) Each group member votes for "red" or "blue", (4) Group decision, (5) Each group member receives earnings according to the group decision, and (6) Each group member receives feedback.

In the beginning of each period, a prize is placed in one of two boxes; a red box and a blue box. It is equally likely that the prize is placed in either box. That is, there is $50 \%$ probability 
that the prize is placed in the blue box and $50 \%$ probability that the prize is placed in the red box. The group's task is to choose a box. Each group member can vote for the box he/she thinks contains the prize. The box that receives the majority of the votes is the group decision. In case of a tie a computer will pick one of the two boxes. There is $50 \%$ probability that either of the two boxes is picked.

Each member of the group earns points as follows:

1. 30 points for each group member if the group finds the prize.

2. -70 points for each group member if the group does not find the prize.

Your earnings are determined exclusively by the group decision. The group decision depends on the votes of all three members. If the group decision is correct, all group members earn 30 points. If the group decision is wrong, all group members earn -70 points. These earnings are independent of how a particular group member voted. Consider the following example. You have voted for the red box and the two other group members both voted for the blue box. This means, that the group decision is the blue box.

1. Suppose the prize was placed in the blue box. Then, each group member, including you, earns 30 points.

2. Now suppose the prize was placed in the red box. Then, each group member, including you, earns -70 points.

The table below illustrates that the only thing influencing your earnings is whether the group finds the prize. The only way you can influence your earnings, is by affecting the decision of the group:

\begin{tabular}{|c|c|c|}
\hline \multirow{4}{*}{$\begin{array}{l}\text { You voted for the correct box } \\
\text { You voted for the wrong box } \\
\text { You did not vote }\end{array}$} & The group is correct & The group is wrong \\
\hline & 30 & -70 \\
\hline & 30 & -70 \\
\hline & 30 & -70 \\
\hline
\end{tabular}

In each period each group member has three options: (1) Vote for the red box, (2) Vote for the blue box, or (3) Abstain (do not cast a vote). 
ABS-VOT \& VOT-ABS: [In the beginning of each period each participant receives information about where the prize is placed. The information participants receive is not $100 \%$ reliable but it is always more likely to be correct than wrong. The participants will not receive equally reliable information; one of the members in a group will receive more reliable information than the other two who get equally reliable information. Reliability refers to how often the information is correct.]

HOM65-ABS \& VOT-VOTB-HOM79: [In the beginning of each period each participant receives information about where the prize is placed. The information participants receive is not $100 \%$ reliable but it is always more likely to be correct than wrong. The participants will not necessarily receive equally reliable information; for example, one of the members in a group can receive more reliable information than the other two who get equally reliable information. Reliability refers to how often the information is correct.]

ABS-VOT \& HOM65-ABS: [For example, in a given period, one member of the group receives information that is correct $90 \%$ of the time, whereas the information that the other two members receive is correct $65 \%$ of the time.]

VOT-ABS \& VOT-VOTB-HOM79: [For example, in a given period, one member of the group receives information that is correct $83 \%$ of the time, whereas the information that the other two members receive is correct $79 \%$ of the time.]

ABS-VOT \& HOM65-ABS: [To illustrate, suppose the prize is placed in the red box. The group member with the most reliable information will receive the information "red" $90 \%$ of the time and "blue" $10 \%$ of the time.]

VOT-ABS \& VOT-VOTB-HOM79: [To illustrate, suppose the prize is placed in the red box. The group member with the most reliable information will receive the information "red" $83 \%$ of the time and "blue" $17 \%$ of the time.]

Your information is personal, that is, it is independent of the other member's information. The two group members with less reliable information do not necessarily get the same informa- 
tion. Suppose you receive information that is correct $65 \%$ of the time, another member of your group also receives information that is correct $65 \%$ and the last member receives information that is correct $90 \%$ of the time. In this case it is possible that you receive the information "red" while the other two members receive the information "blue". It is randomly decided at the beginning of each period who gets which type of information. The reliability of the information can change during the experiment, in which case you will be informed.

[Subjects are shown the feedback screen.] After each period, all group members receive feedback as follows: (1) The reliability of each group member's information and their choice (red, blue or abstain), (2) The outcome of the period; that is, whether the group decision was correct or not, and (3) The history of results in periods with different number of voters. That is, the number of periods with different number of voters and the corresponding average earnings. Do you have any questions?

\section{Appendix B: Multinomial Logit Estimates by Treatment}

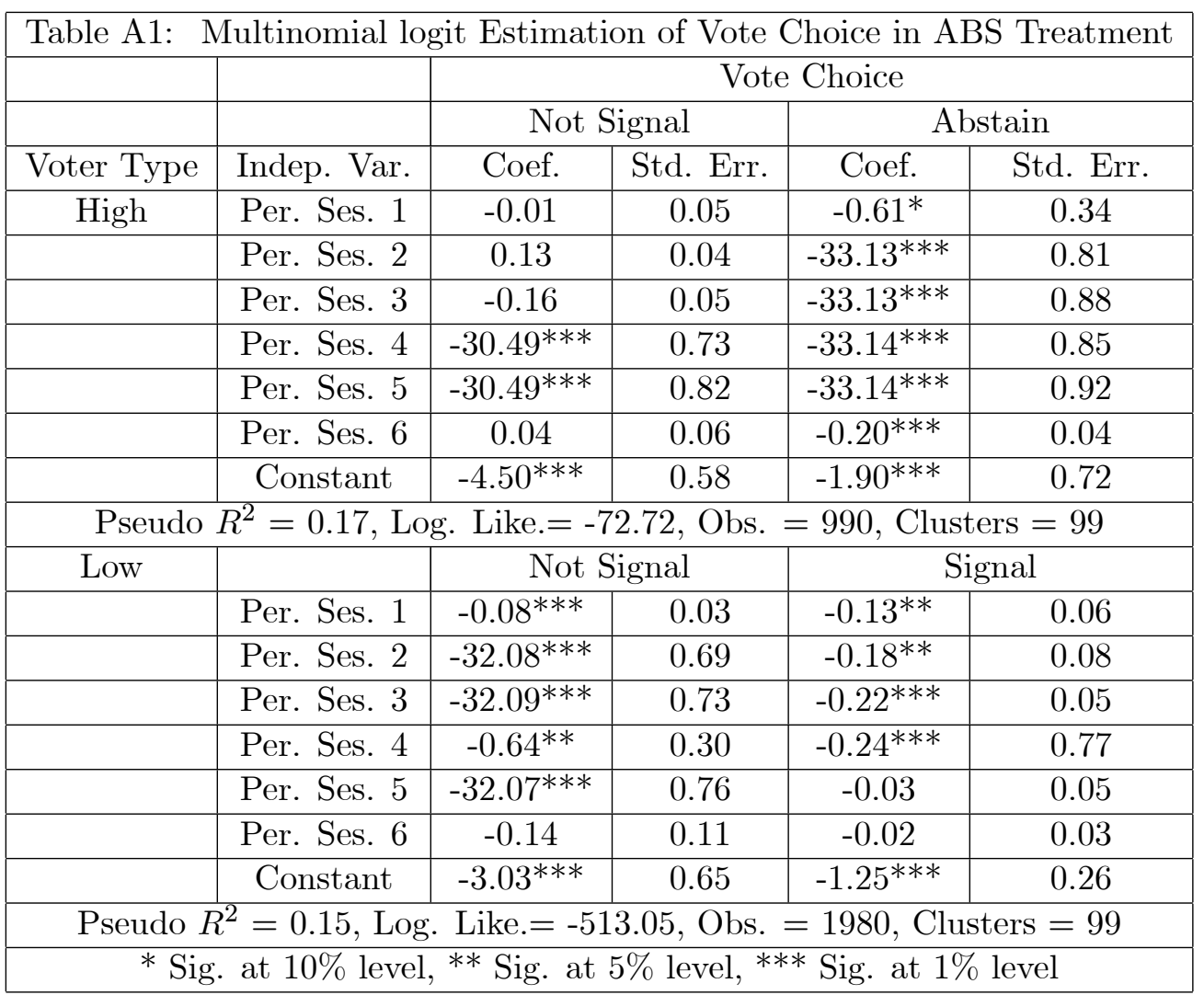




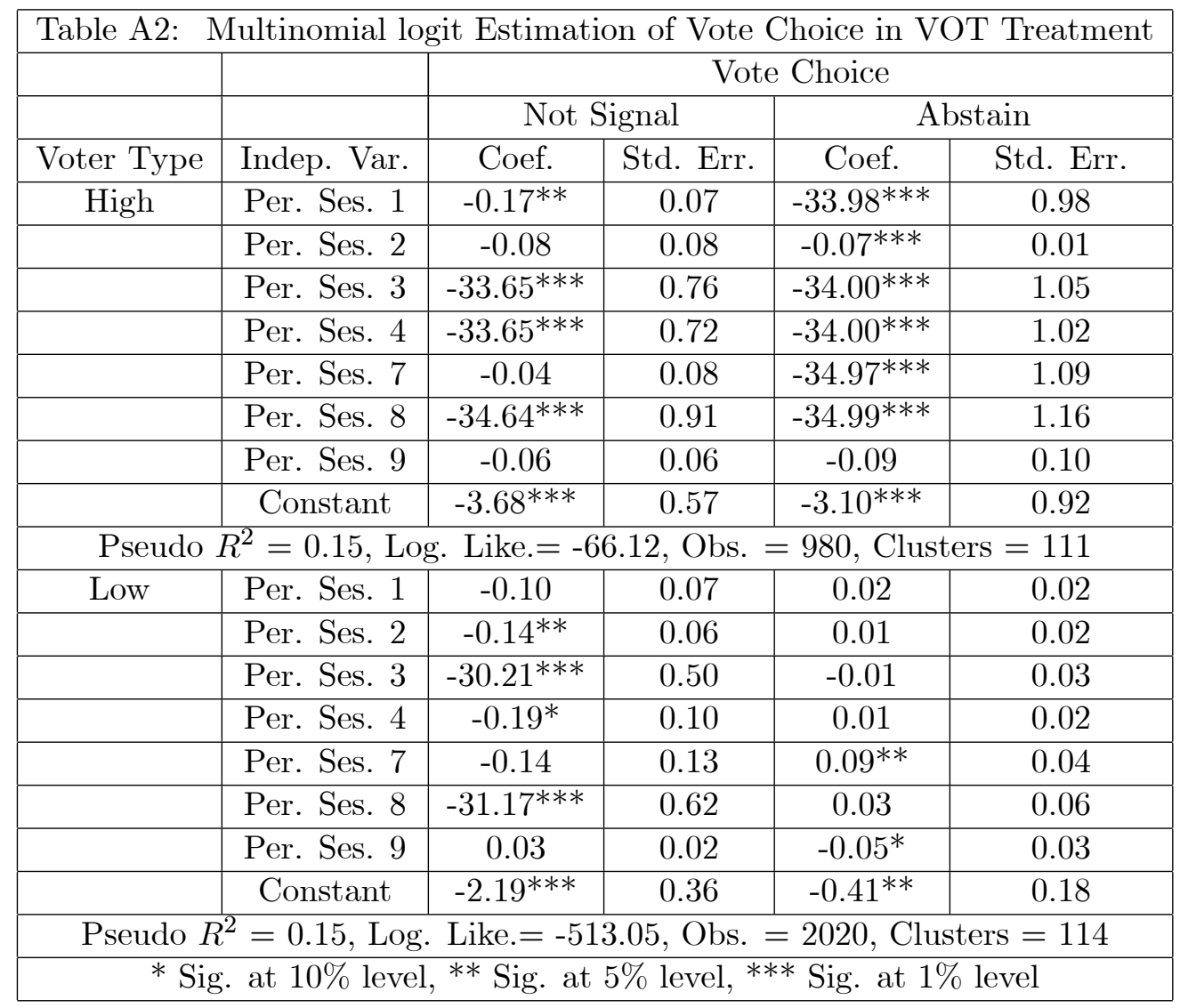




\begin{tabular}{|c|c|c|c|c|c|}
\hline & & \multicolumn{4}{|c|}{ Vote Choice (VOTB Treatment) } \\
\hline & & \multicolumn{2}{|c|}{ Not Signal } & \multicolumn{2}{|c|}{ Abstain } \\
\hline Voter Type & Indep. Var. & Coef. & Std. Err. & Coef. & Std. Err. \\
\hline \multirow[t]{4}{*}{ High } & Per. Ses. 7 & $-37.80^{* * *}$ & 0.58 & -0.05 & 0.11 \\
\hline & Per. Ses. 8 & $-37.83^{* * *}$ & 0.65 & $-37.68^{* * *}$ & 0.88 \\
\hline & Per. Ses. 9 & 0.01 & 0.03 & 0.01 & 0.05 \\
\hline & Constant & $-2.97 * * *$ & 0.41 & $-3.65^{* * *}$ & 0.72 \\
\hline \multicolumn{6}{|c|}{ Pseudo $R^{2}=0.08$, Log. Like. $=-123.07$, Obs. $=560$, Clusters $=42$} \\
\hline \multirow[t]{4}{*}{ Low } & Per. Ses. 7 & $-33.28^{* * *}$ & 0.79 & 0.05 & 0.05 \\
\hline & Per. Ses. 8 & $-33.27^{* * *}$ & 0.88 & -0.10 & 0.08 \\
\hline & Per. Ses. 9 & -0.04 & 0.04 & -0.4 & 0.04 \\
\hline & Constant & $-1.33^{* *}$ & 0.55 & -0.42 & 0.35 \\
\hline \multicolumn{6}{|c|}{ Pseudo $R^{2}=0.08$, Log. Like. $=-220.07$, Obs. $=280$, Clusters $=42$} \\
\hline & \multicolumn{5}{|c|}{ Vote Choice (HOM 65 Treatment) } \\
\hline \multirow[t]{3}{*}{ All } & Per. Ses. 5 & $0.06^{* * *}$ & 0.02 & -0.01 & 0.03 \\
\hline & Per. Ses. 6 & 0.00 & 0.04 & -0.03 & 0.02 \\
\hline & Constant & $-3.70^{* * *}$ & 0.57 & $-2.25^{* * *}$ & 0.41 \\
\hline \multicolumn{6}{|c|}{ Pseudo $R^{2}=0.02$, Log. Like. $=-320.21$, Obs. $=810$, Clusters $=27$} \\
\hline & \multicolumn{5}{|c|}{ Vote Choice ;(HOM79 Treatment) } \\
\hline \multirow[t]{4}{*}{ All } & Per. Ses. 7 & -0.10 & 0.09 & -0.03 & 0.06 \\
\hline & Per. Ses. 8 & $-31.85^{* * *}$ & 0.55 & $-0.49^{* *}$ & 0.23 \\
\hline & Per. Ses. 9 & -0.00 & 0.02 & 0.00 & 0.03 \\
\hline & Constant & $-2.43^{* * *}$ & 0.36 & $-2.49^{* * *}$ & 0.41 \\
\hline \multicolumn{6}{|c|}{ Pseudo $R^{2}=0.05$, Log. Like. $=-347.20$, Obs. $=840$, Clusters $=42$} \\
\hline & * Sig. at 10 & 0 level, **s & ig. at $5 \%$ & vel, *** Si & evel \\
\hline
\end{tabular}




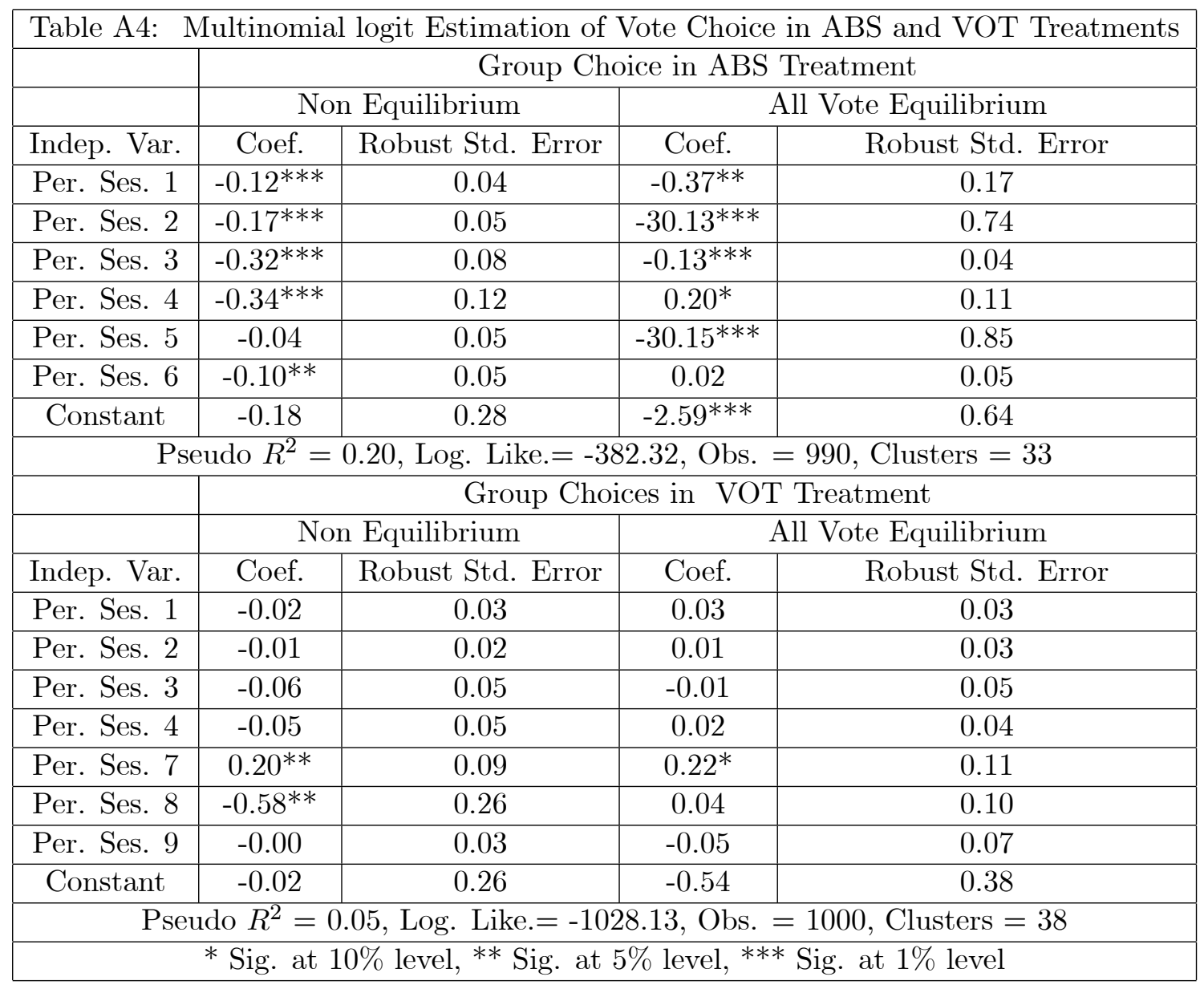




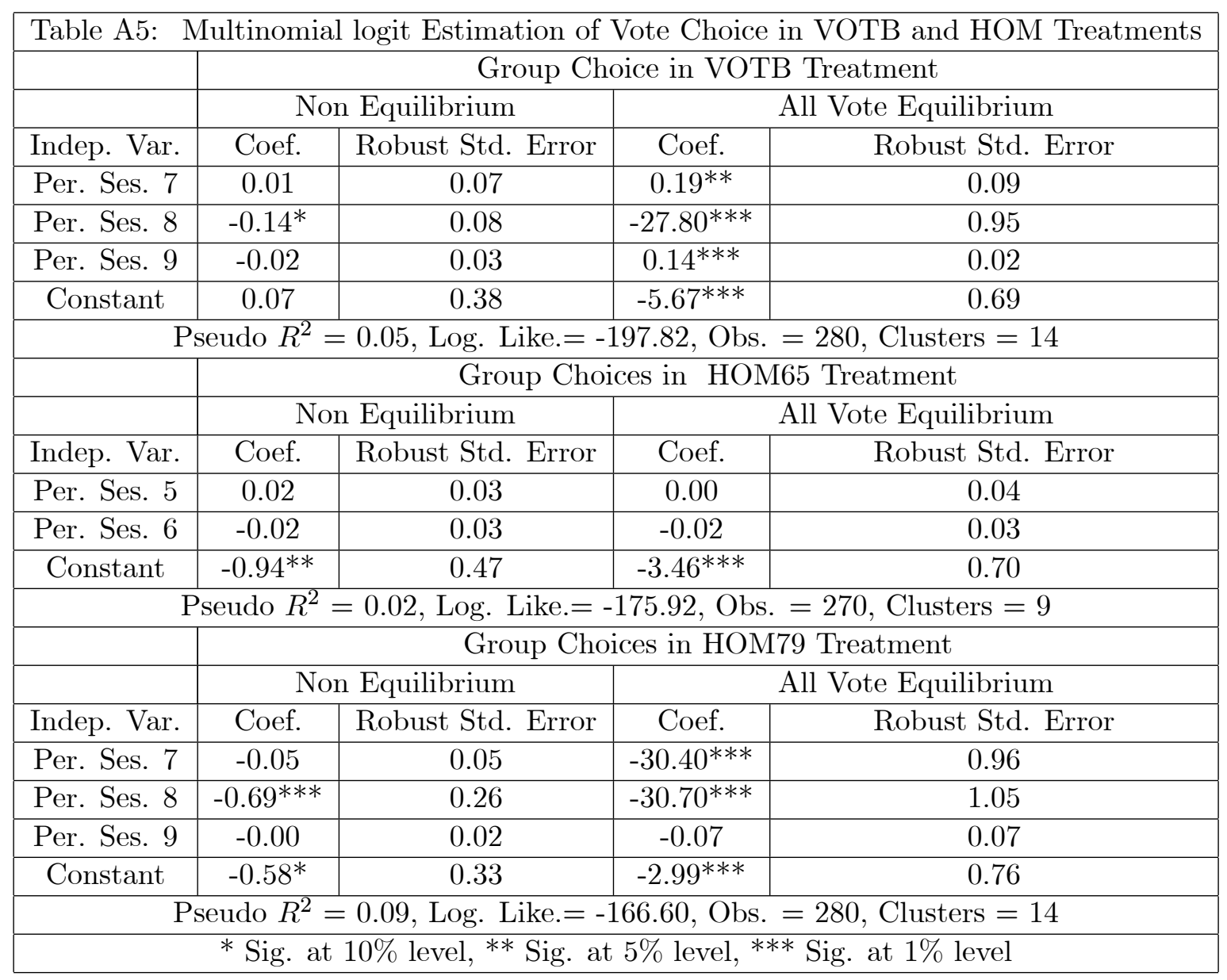

\section{References}

[1] Ali, S. Nageeb, Jacob K. Goeree, Navin Kartik, and Thomas R. Palfrey. 2008. "Information Aggregation in Standing and Ad Hoc Committees," American Economic Review: Papers and Proceedings 98(May, 2):181-186.

[2] Austen-Smith, David and Jeffrey Banks. 1996. "Information Aggregation, Rationality, and the Condorcet Jury Theorem," American Political Science Review 90(1):34-45.

[3] Battaglini, Marco, Rebecca B. Morton, and Thomas R. Palfrey. 2008a. "Information Aggregation and Strategic Abstention in Large Laboratory Elections," American Economic Review: Papers and Proceedings 98(May, 2):194-200. 
[4] Battaglini, Marco, Rebecca B. Morton, and Thomas R. Palfrey. 2008b. "The Swing Voter's Curse in the Laboratory," working paper, New York University.

[5] Canes-Wrone, Brandice, Michael C. Herron, and Kenneth W. Shotts. 2001. "Leadership and Pandering: A Theory of Executive Policymaking." American Journal or Political Science 45(July):532-550.

[6] Clark, Kenneth and Martin Sefton. 2001. "Repetition and Signalling: Experimental Evidence from Games with Efficient Equilibria," Economic Letters 70:357-362.

[7] Condorcet, Marie Jean Antoine Nicolas de Caritat marquis de. 1785. Essai sur l'application de l'analyse à la probabilité des decisions rendues à la pluralité des voix. Paris, France: Imprimerie Royale.

[8] Devetag, Giovanna and Andreas Ortmann. 2007. "When and Why? A Critical Survey on Coordination Failure in the Laboratory," Experimental Economics 10:331-344.

[9] Feddersen, Timothy and Wolfgang Pesendorfer. 1996. "The Swing Voter's Curse," American Economic Review, 86(3):408-24.

[10] Feddersen, Timothy and Wolfgang Pesendorfer. 1998. "Convicting the Innocent: The Inferiority of Unanimous Jury Verdicts," American Political Science Review 92:23-35.

[11] Feddersen, Timothy J. and Pesendorfer, Wolfgang. 1999. "Abstention in Elections with Asymmetric Information and Diverse Preferences." American Political Science Review, 93 (2): 381398 .

[12] Fischbacher, Urs. 2007. "Zurich Toolbox for Readymade Economic Experiments," Experimental Economics 10(2):171-8.

[13] Greiner, B. 2004. "An Online Recruitment System for Economic Experiments," in Kurt Kremer and Volker Mecho (eds..), Forschung und Wissenschaftliches Rechnen. GWDG Bericht 63:79-93. 
[14] McMurray, Joseph C. 2008. "Information and Voting: The Wisdom of the Experts versus the Wisdom of the Masses," working paper, University of Rochester.

[15] Meirowitz, Adam. 2002. "Informative Voting and Condorcet Jury Theorems with a Continuum of Types," Social Choice and Welfare. 19:219-236. 